\title{
Covering of the null ideal may have countable cofinality
}

\author{
by
}

Saharon Shelah (Jerusalem and New Brunswick, NJ)

\begin{abstract}
We prove that it is consistent that the covering number of the ideal of measure zero sets has countable cofinality.
\end{abstract}

0. Introduction. In the present paper we show that it is consistent that the covering of the null ideal has countable cofinality. Recall that the covering number of the null ideal (i.e. the ideal of measure zero sets) is defined as

$$
\operatorname{cov}(\text { null })=\min \left\{|\mathcal{P}|: \mathcal{P} \subseteq \text { null and } \bigcup_{A \in \mathcal{P}} A=\mathbb{R}\left(={ }^{\omega_{2}}\right)\right\} .
$$

The question whether the cofinality of $\operatorname{cov}($ null) is uncountable has been raised by D. Fremlin and has been around since the late seventies. It appears in Fremlin's list of problems, [Fe94], as problem CO. Recall that for the ideal of meagre sets the answer is positive: A. Miller [Mi82] proved that the cofinality of the covering of category is uncountable. T. Bartoszyński [Ba88] saw that $\mathfrak{b}<\aleph_{\omega}$ is necessary (see [BaJu95, Ch. 5] for more results related to this problem). It should be noted that most people thought $\operatorname{cf}(\operatorname{cov}($ null $))=\aleph_{0}$ was impossible.

The main result of this paper is the following:

TheOREM 0.1. Con $\left(\operatorname{cov}(\right.$ null $\left.)=\aleph_{\omega}+\mathrm{MA}_{\aleph_{n}}\right)$ for each $n<\omega$.

The presentation of the proof of 0.1 sacrifices generality for hopeful transparency. We finish by some further remarks, e.g. the exact cardinal assumption for 0.1 . We try to make the paper self-contained for readers with basic knowledge of forcing.

2000 Mathematics Subject Classification: 03E17, 03E35.

Key words and phrases: null sets, cardinal invariants of the continuum, iterated forcing, ccc forcing.

Research supported by "The Israel Science Foundation" founded by The Israel Academy of Sciences and Humanities. Publication no. 592. 
In a subsequent paper, [Sh 619], we deal with the question: "can every non-null set be partitioned into uncountably many non-null sets", equivalently: "can the ideal of null sets which are subsets of a non-null subset of $\mathbb{R}$ be $\aleph_{1}$-saturated". P. Komjáth [Ko89] proved that it is consistent that there is a non-meagre set $A$ such that the ideal of meagre subsets of $A$ is $\aleph_{1}$-saturated. The question whether a similar fact may hold for measure dates back to Ulam; see also Prikry's thesis. It appears as question $E L(a)$ on Fremlin's list. In [Sh 619] we prove the following:

THEOREM 1. It is consistent that there is a non-null set $A \subseteq \mathbb{R}$ such that the ideal of null subsets of $A$ is $\aleph_{1}$-saturated (of course, provided that " $Z F C$ $+\exists$ measurable" is consistent).

In [Sh 619] we also prove the following.

THEOREM 2. It is consistent that:

$(\oplus) \quad$ there is a non-null $A \subseteq \mathbb{R}$ such that for every $f: A \rightarrow \mathbb{R}$, the function $f$ as a subset of the plane $\mathbb{R} \times \mathbb{R}$ is null

provided that " $Z F C+$ there is a measurable cardinal" is consistent.

Notation 0.2. We denote:

- natural numbers by $k, l, m, n$ and also $i, j$,

- ordinals by $\alpha, \beta, \gamma, \delta, \zeta, \xi$ ( $\delta$ always limit),

- cardinals by $\lambda, \kappa, \chi, \mu$,

- reals by $a, b$ and positive reals (normally small) by $\varepsilon$,

- subsets of $\omega$ or ${ }^{\omega \geq 2}$ or Ord by $A, B, C, X, Y, Z$ but $\mathcal{B}$ is a Borel function,

- finitely additive measures by $\Xi$,

- sequences of natural numbers or ordinals by $\eta, \nu, \varrho$,

$s$ is used for various things, $\mathcal{T}$ is as in Definition 2.9, $t$ is a member of $\mathcal{T}$. We denote

- forcing notions by $P, Q$,

- forcing conditions by $p, q$

and use $r$ to denote members of Random (see below) except in Definition 2.2.

Leb is the Lebesgue measure (on ${ }^{\omega} 2$ ), and Random will be the family

$\left\{r \subseteq{ }^{\omega>} 2: r\right.$ is a subtree of $(\omega>2, \triangleleft)$ (i.e. non-empty subset of ${ }^{\omega>} 2$ closed under initial segments) with no $\triangleleft$-maximal element (so $\lim (r):=\left\{\eta \in \omega^{\omega} 2:(\forall n \in \omega)(\eta\lceil n \in t)\}\right.$ is a closed subset of $\left.\omega_{2}\right)$ and $\left.\operatorname{Leb}(\lim (r))>0\right\}$

ordered by inverse inclusion. We may sometimes use instead

$\left\{B: B\right.$ is a Borel non-null subset of $\left.{ }^{\omega} 2\right\}$. 
For $\eta \in{ }^{\omega>} 2$ and $A \subseteq{ }^{\omega \geq} 2$ let

$$
A^{[\eta]}=\{\nu \in A: \nu \unlhd \eta \vee \eta \unlhd \nu\} .
$$

Let $\mathcal{H}(\chi)$ denote the family of sets with transitive closure of cardinality $<\chi$, and let $<_{\chi}^{*}$ denote a well ordering of $\mathcal{H}(\chi)$.

We thank Tomek Bartoszyński and Mariusz Rabus for reading and commenting and correcting.

1. Preliminaries. We review various facts on finitely additive measures.

Definition 1.1. (1) $\mathcal{M}$ is the set of functions $\Xi$ from some Boolean subalgebra $P$ of $\mathcal{P}(\omega)$ including the finite sets to $[0,1]_{\mathbb{R}}$ such that:

- $\Xi(\emptyset)=0, \Xi(\omega)=1$,

- if $Y, Z \in P$ are disjoint, then $\Xi(Y \cup Z)=\Xi(Y)+\Xi(Z)$,

- $\Xi(\{n\})=0$ for $n \in \omega$.

(2) $\mathcal{M}^{\text {full }}$ is the set of $\Xi \in \mathcal{M}$ with domain $\mathcal{P}(\omega)$ and members are called finitely additive measures (on $\omega$ ).

(3) We say $A$ has $\Xi$-measure $a$ (or $>a$, or whatever) if $A \in \operatorname{dom}(\Xi)$ and $\Xi(A)$ is $a$ (or $>a$, or whatever).

Proposition 1.2. Let $a_{\alpha}, b_{\alpha}\left(\alpha<\alpha^{*}\right)$ be reals, $0 \leq a_{\alpha} \leq b_{\alpha} \leq 1$, and let $A_{\alpha} \subseteq \omega\left(\alpha<\alpha^{*}\right)$ be given. The following conditions are equivalent:

(A) There exists $\Xi \in \mathcal{M}$ which satisfies $\Xi\left(A_{\alpha}\right) \in\left[a_{\alpha}, b_{\alpha}\right]$ for $\alpha<\alpha^{*}$.

(B) For every $\varepsilon>0, m<\omega$ and $n<\omega$, and $\alpha_{0}<\alpha_{1}<\ldots<\alpha_{n-1}<\alpha^{*}$ we can find a finite, non-empty $u \subseteq[m, \omega)$ such that for $l<n$,

$$
a_{\alpha_{l}}-\varepsilon \leq\left|A_{\alpha_{l}} \cap u\right| /|u| \leq b_{\alpha_{l}}+\varepsilon .
$$

(C) For every real $\varepsilon>0, n<\omega$ and $\alpha_{0}<\alpha_{1}<\ldots<\alpha_{n-1}<\alpha^{*}$ there are $c_{l} \in\left[a_{\alpha_{l}}-\varepsilon, b_{\alpha_{l}}+\varepsilon\right]$ such that in the vector space $\mathbb{R}^{n},\left\langle c_{0}, \ldots, c_{n-1}\right\rangle$ is in the convex hull of $\left\{\varrho \in{ }^{n}\{0,1\}\right.$ : for infinitely many $m \in \omega$ we have $\left.(\forall l<n)\left[\varrho(l)=1 \Leftrightarrow m \in A_{\alpha_{l}}\right]\right\}$.

(D) Like part (A) with $\Xi \in \mathcal{M}^{\text {full }}$.

(E) Like part (B) demanding $u \subseteq \omega,|u| \geq m$.

P r o o f. Straightforward (in fact, in clause (E) we can omit " $u \subseteq[m, \omega)$ "). On (C) see 2.17.

Proposition 1.3. (1) Assume that $\Xi_{0} \in \mathcal{M}$ and for $\alpha<\alpha^{*}, A_{\alpha} \subseteq \omega$ and $0 \leq a_{\alpha} \leq b_{\alpha} \leq 1, a_{\alpha}, b_{\alpha}$ reals. The following are equivalent:

(A) There is $\Xi \in \mathcal{M}^{\text {full }}$ extending $\Xi_{0}$ such that $\alpha<\alpha^{*} \Rightarrow \Xi\left(A_{\alpha}\right) \in$ $\left[a_{\alpha}, b_{\alpha}\right]$.

(B) For every partition $\left\langle B_{0}, \ldots, B_{m-1}\right\rangle$ of $\omega$ with $B_{i} \in \operatorname{dom}\left(\Xi_{0}\right)$ and $\varepsilon>0, n<\omega$ and $\alpha_{0}<\ldots<\alpha_{n-1}<\alpha^{*}$ we can find a finite set $u \subseteq \omega$ such that $\Xi\left(B_{i}\right)-\varepsilon \leq\left|u \cap B_{i}\right| /|u| \leq \Xi\left(B_{i}\right)+\varepsilon$ and $a_{\alpha_{l}}-\varepsilon \leq\left|u \cap A_{\alpha_{l}}\right| /|u| \leq b_{\alpha_{l}}+\varepsilon$. 
(C) For every partition $\left\langle B_{0}, \ldots, B_{m-1}\right\rangle$ of $\omega$ with $B_{i} \in \operatorname{dom}\left(\Xi_{0}\right)$ and $\varepsilon>0, n<\omega$ and $\alpha_{0}<\ldots<\alpha_{n-1}<\alpha^{*}$ we can find $c_{l, k} \in[0,1]_{\mathbb{R}}$ for $l<n, k<m$ such that:

(a) $\sum_{k<m} c_{l, k} \in\left(a_{\alpha_{l}}-\varepsilon, b_{\alpha_{l}}+\varepsilon\right)$,

(b) for each $k<m$ and $s<\omega$ we can find $u \subseteq B_{k}$ with $\geq s$ members such that

$$
l<n \Rightarrow c_{l, k}-\varepsilon<\left(\left|u \cap A_{l} \cap B_{k}\right| /|u|\right) \Xi\left(B_{k}\right)<c_{l, k}+\varepsilon .
$$

(D) for every partition $\left\langle B_{0}, \ldots, B_{m-1}\right\rangle$ of $\omega$ with $B_{i} \in \operatorname{dom}\left(\Xi_{0}\right), \varepsilon>0$, $n<\omega$, and $\alpha_{0}, \ldots, \alpha_{n-1}<\alpha^{*}$ we can find $c_{l, k} \in[0,1]_{\mathbb{R}}$ for $l<n, k<m$ such that:

(a) $\sum_{k<m} c_{l, k} \in\left[a_{\alpha_{l}}-\varepsilon, b_{\alpha_{l}}+\varepsilon\right]$,

(b) $\left\langle c_{l, k}: l<n\right\rangle$ is in the convex hull of

$$
\begin{gathered}
\left\{\varrho \in{ }^{n}\{0,1\}: \text { for infinitely many } i \in B_{k},\right. \text { we have } \\
\left.(\forall l<n)\left[\varrho(l)=1 \Leftrightarrow i \in A_{\alpha_{l}}\right]\right\} .
\end{gathered}
$$

(2) The following are sufficient conditions for (A)-(D) above:

(E) For every $\varepsilon>0, A^{*} \in \operatorname{dom}\left(\Xi_{0}\right)$ such that $\Xi_{0}\left(A^{*}\right)>0, n<\omega$, $\alpha_{0}<\ldots<\alpha_{n-1}<\alpha^{*}$, we can find a finite, non-empty $u \subseteq A^{*}$ such that $a_{\alpha_{l}}-\varepsilon \leq\left|A_{\alpha_{l}} \cap u\right| /|u| \leq b_{\alpha_{l}}+\varepsilon$ for $l<n$.

(F) For every $\varepsilon>0, n<\omega, \alpha_{0}<\alpha_{1}<\ldots<\alpha_{n-1}<\alpha^{*}$ and $A^{*} \in$ $\operatorname{dom}\left(\Xi_{0}\right)$ such that $\Xi_{0}\left(A^{*}\right)>0$, the set $\prod_{l<n}\left[a_{\alpha_{l}}-\varepsilon, b_{\alpha_{l}}+\varepsilon\right] \subseteq \mathbb{R}^{n}$ is not disjoint from the convex hull of

$$
\begin{aligned}
\left\{\varrho \in{ }^{n}\{0,1\}: \text { for infinitely many } m \in A^{*}\right. \text { we have } \\
\\
\left.(\forall l<n)\left[\varrho(l)=1 \Leftrightarrow m \in A_{\alpha_{l}}\right]\right\} .
\end{aligned}
$$

(3) If in addition $b_{\alpha}=1$ for $\alpha<\alpha^{*}$ then a sufficient condition for (A)-(E) above is

(G) if $A^{*} \in \operatorname{dom}\left(\Xi_{0}\right)$ and $\Xi\left(A^{*}\right)>0$ and $n<\omega$ and $\alpha_{0}<\ldots<\alpha_{n-1}<$ $\alpha^{*}$ then $A^{*} \cap \bigcap_{l<n} A_{\alpha_{l}} \neq \emptyset$.

Pr o of. Straightforward.

Definition 1.4. (1) For $\Xi \in \mathcal{M}^{\text {full }}$ and a sequence $\bar{a}=\left\langle a_{l}: l<\omega\right\rangle$ of reals in $[0,1]_{\mathbb{R}}$ (or just $\sup _{l<\omega}\left|a_{l}\right|<\infty$ ), define $\operatorname{Av} \Xi(\bar{a})$ to be $\sup \left\{\sum_{k<k^{*}} \Xi\left(A_{k}\right) \inf \left\{a_{l}: l \in A_{k}\right\}:\left\langle A_{k}: k<k^{*}\right\rangle\right.$ is a partition of $\left.\omega\right\}$ $=\inf \left\{\sum_{k<k^{*}} \Xi\left(A_{k}\right) \sup \left\{a_{l}: l \in A_{k}\right\}:\left\langle A_{k}: k<k^{*}\right\rangle\right.$ is a partition of $\left.\omega\right\}$.

(Easily proved that they are equal.) 
(2) For $\Xi \in \mathcal{M}$ and $A \subseteq \omega$ such that $\Xi(A)>0$ we define

$$
\Xi_{A}(B)=\Xi(A \cap B) / \Xi(A) \text {. }
$$

Clearly $\Xi_{A} \in \mathcal{M}$ with the same domain, $\Xi_{A}(A)=1$. If $B \subseteq \omega$ and $\Xi(B)>0$ then we let

$$
\operatorname{Av}_{\Xi\lceil B}\left(\left\langle a_{k}: k \in B\right\rangle\right)=\operatorname{Av}_{\Xi}\left(\left\langle a_{k}^{\prime}: k<\omega\right\rangle\right) / \Xi(B)
$$

where

$$
a_{k}^{\prime}= \begin{cases}a_{k} & \text { if } k \in B \\ 0 & \text { if } k \notin B .\end{cases}
$$

Proposition 1.5. Assume $\Xi \in \mathcal{M}^{\text {full }}$ and $a_{l}^{i} \in[0,1]_{\mathbb{R}}$ for $i<i^{*}<\omega$, $l<\omega, B \subseteq \omega, \Xi(B)>0$ and $\operatorname{Av}_{\Xi_{B}}\left(\left\langle a_{l}^{i}: l<\omega\right\rangle\right)=b_{i}$ for $i<i^{*}, m^{*}<\omega$ and lastly $\varepsilon>0$. Then for some finite $u \subseteq B \backslash m^{*}$ we have: if $i<i^{*}$, then $b_{i}-\varepsilon<\sum\left\{a_{l}^{i}: l \in u\right\} /|u|<b_{i}+\varepsilon$.

Proof. Let $B=\bigcup_{j<j^{*}} B_{j}$ be a partition of $B$ with $j^{*}<\omega$ such that for every $i<i^{*}$ we have

$$
\sum_{j<j^{*}} \sup \left\{a_{l}^{i}: l \in B_{j}\right\} \Xi\left(B_{j}\right)-\sum_{j<j^{*}} \inf \left\{a_{l}^{i}: l \in B_{j}\right\} \Xi\left(B_{j}\right)<\varepsilon / 2 .
$$

Now choose $k^{*}$ large enough such that there are $k_{j}$ satisfying $k^{*}=\sum_{j<j^{*}} k_{j}$ and $\left|k_{j} / k^{*}-\Xi\left(B_{j}\right) / \Xi(B)\right|<\varepsilon /\left(2 j^{*}\right)$ for $j<j^{*}$. Let $u_{j} \subseteq B_{j} \backslash m^{*}$ with $\left|u_{j}\right|=k_{j}$ for $j<j^{*}$, and let $u=\bigcup_{j<j^{*}} u_{j}$. Now calculate:

$$
\begin{aligned}
\sum_{l \in u} a_{l}^{i} /|u| & =\sum_{j<j^{*}} \sum\left\{a_{l}^{i}: l \in u_{j}\right\} /|u| \leq \sum_{j<j^{*}} \sup \left\{a_{l}^{i}: l \in B_{j}\right\} k_{j} / k^{*} \\
& \leq \sum_{j<j^{*}} \sup \left\{a_{l}^{i}: l \in B_{j}\right\}\left(\Xi\left(B_{j}\right) / \Xi(B)+\varepsilon /\left(2 j^{*}\right)\right) \\
& \leq b_{i}+\varepsilon / 2+\varepsilon / 2=b_{i}+\varepsilon, \\
\sum_{l \in u} a_{l}^{i} /|u| & =\sum_{j<j^{*}} \sum\left\{a_{l}^{i}: l \in u_{j}\right\} /|u| \geq \sum_{j<j^{*}} \inf \left\{a_{l}^{i}: l \in B_{j}\right\} k_{j} / k^{*} \\
& \geq \sum_{j<j^{*}} \inf \left\{a_{l}^{i}: l \in B_{j}\right\}\left(\Xi\left(B_{j}\right) / \Xi(B)-\varepsilon /\left(2 j^{*}\right)\right)>b_{i}-\varepsilon .
\end{aligned}
$$

Claim 1.6. Suppose $Q_{1}, Q_{2}$ are forcing notions, $\Xi_{0} \in \mathcal{M}^{\text {full }}$ in $V$, and for $l=1,2$,

$$
\Vdash_{Q_{l}} \stackrel{\Xi_{l}}{\sim} \text { is a finitely additive measure extending } \Xi_{0} " .
$$

Then

$\Vdash_{Q_{1} \times Q_{2}}$ "there is a finitely additive measure extending $\underset{\sim}{\Xi_{1}}$ and $\underset{\sim}{\Xi_{2}}$

(hence $\left.\Xi_{0}\right)$ ". 
Proof. Straightforward by 1.2 as

(*) if $\Vdash_{Q_{l}}$ " ${\underset{\sim}{l}}_{l} \subseteq \omega$ " and $\Vdash_{Q_{1} \times Q_{2}}$ " ${\underset{\sim}{1}}_{1} \cap \underset{\sim}{A_{2}}$ is finite" then

$\Vdash_{Q_{1} \times Q_{2}}$ "for some $m$ and $A \subseteq \omega, A \in V$ we have

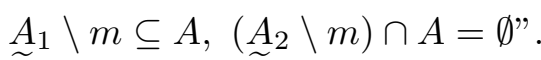

FACT 1.7. Assume $\Xi$ is a partial finitely additive measure, and $\bar{a}^{\alpha}=$ $\left\langle a_{k}^{\alpha}: k<\omega\right\rangle$ a sequence of reals for $\alpha<\alpha^{*}$ such that $\lim \sup _{k}\left|a_{k}^{\alpha}\right|<\infty$ for each $\alpha$. Then $(\mathrm{B}) \Rightarrow(\mathrm{A})$ where

(A) there is $\Xi^{*}$ with $\Xi \subseteq \Xi^{*} \in \mathcal{M}^{\text {full }}$ such that $\operatorname{Av}_{\Xi^{*}}\left(\bar{a}^{\alpha}\right) \geq b_{\alpha}$ for $\alpha<\alpha^{*}$,

(B) for every partition $\left\langle B_{0}, \ldots, B_{m^{*}-1}\right\rangle$ of $\omega$ with $B_{m} \in \operatorname{dom}(\Xi)$ and $\varepsilon>0, k^{*}>0$ and $\alpha_{0}<\ldots<\alpha_{n^{*}-1}<\alpha^{*}$, there is a finite $u \subseteq \omega \backslash k^{*}$ such that:

(i) $\Xi\left(B_{m}\right)-\varepsilon<\left|u \cap B_{m}\right| /|u|<\Xi\left(B_{m}\right)+\varepsilon$,

(ii) $|u|^{-1} \sum_{k \in u} a_{k}^{\alpha_{l}}>b_{\alpha_{l}}-\varepsilon$ for $l<n^{*}$.

REMARK 1.8. If in (A) we demand $\operatorname{Av}_{\Xi}\left(\bar{a}^{\alpha}\right)=b_{\alpha}$, then in (B)(ii) add $|u|^{-1} \sum_{k \in u} a_{k}^{\alpha_{l}} \leq b_{\alpha_{l}}+\varepsilon$.

2. The iteration. Ignoring $\mathrm{MA}_{<\kappa}$ (which anyhow was a side issue) a quite natural approach in order to get 0.1 (i.e. $\operatorname{cov}($ null $)=\lambda$, say $\lambda=\aleph_{\omega}$ ) is to use finite support iteration, $\bar{Q}=\left\langle P_{\alpha}, Q_{\alpha}: \alpha<\alpha^{*}\right\rangle$, add in the first $\lambda$ steps null sets $N_{\alpha}$ (the intention is that $\bigcup_{\alpha<\lambda} N_{\alpha}={ }^{\omega} 2$ in the final model), and then iterate with $Q_{\alpha}$ being Random $\mathbf{V}^{P_{\alpha}^{\prime}}$ where $P_{\alpha}^{\prime}<P_{\alpha}$ and $\left|P_{\alpha}^{\prime}\right|<\lambda$. Say, for some $A_{\alpha} \subseteq \alpha$,

$$
\begin{array}{r}
P_{\alpha}^{\prime}=\left\{p \in P_{\alpha}: \operatorname{dom}(p) \subseteq A_{\alpha}\right. \text { and this holds for the conditions } \\
\text { involved in the } \left.P_{\gamma} \text {-name } p(\gamma) \text { for } \gamma \in \operatorname{dom}(p) \text { etc. }\right\}
\end{array}
$$

(so each $Q_{\alpha}$ is a partial random; see Definition 2.2). If every set of $<\lambda$ null sets from $\mathbf{V}^{P_{\alpha^{*}}}$ is included in some $\mathbf{V}^{P_{\alpha}^{\prime}}$, clearly $\mathbf{V}^{P_{\alpha^{*}}}=\operatorname{cov}($ null $) \geq \lambda$; but we need the other inequality too. So we are using "non-transitive" memory, i.e. $\alpha \in A_{\beta} \nRightarrow A_{\alpha} \subseteq A_{\beta}$; this makes our life hard.

The problem is: why does $\left\langle N_{\alpha}: \alpha<\lambda\right\rangle$ continue to cover? For $P_{\lambda+n}$ such that $\alpha \in[\lambda, \lambda+n) \Rightarrow A_{\alpha}=\alpha$ this is very clear (we get iteration of Random forcing) and if $\alpha \in[\lambda, \lambda+n) \Rightarrow A_{\alpha} \subseteq \lambda$ this is clear (we get product). But necessarily we get a quite chaotic sequence $\left\langle A_{\alpha_{m}} \cap\left\{\alpha_{l}: l<m\right\}: m<m^{*}\right\rangle$ for some $\alpha_{0}<\ldots<\alpha_{m^{*}-1}$. More concretely this is the problem of why there are no perfect sets of random reals (see 2.7) or even just no dominating reals. We need to "let the partial randoms whisper secrets to one another", in other words to pass information in some way. This is done by the finitely additive measures $\Xi_{\alpha}^{t}$. We had tried with thinking of using $\aleph_{\varepsilon}$-support (see [Sh 538]), the idea is still clear in the proof of 3.3. In this proof we start 
with "no dominating reals" for which we can just use ultrafilters (rather than finitely additive measures).

Let us start with a ground model $\mathbf{V}$ satisfying the following hypothesis:

Hypothesis 2.1. (a) $\lambda=\sum_{\zeta<\delta(*)} \lambda_{\zeta}, \aleph_{0}<\kappa=\operatorname{cf}(\kappa), \kappa<\lambda_{\zeta}<\lambda_{\gamma}$ for $\zeta<\gamma<\delta(*), 2^{\kappa}=\chi$ and $\zeta<\delta(*) \Rightarrow\left(\lambda_{\zeta}\right)^{\aleph_{0}}<\lambda$.

(b) We have one of the following $\left({ }^{1}\right)$ :

$(\alpha) \operatorname{cf}(\chi)>\lambda$, the length of the final iteration is $\chi$,

$(\beta)$ the length of the final iteration is $\chi \times \chi \times \lambda^{+}$and $\operatorname{cf}\left([\chi]^{<\lambda}, \subseteq\right)=\chi$.

We speak mainly on $(\alpha)$. In case $(\beta)$ we should be careful to have no repetitions in $\bar{\eta}=\left\langle\eta_{\alpha}: \alpha<\delta^{*}\right\rangle$ (see below) or $\left\langle\eta_{\alpha} / \approx_{\kappa}: \alpha<\delta^{*}\right\rangle$ with no repetitions, where $\eta \approx_{\kappa} \nu$ iff $\eta, \nu \in{ }^{\kappa} 2$ and $|\{i<\kappa: \eta(i) \neq \nu(i)\}|<\kappa$.

The reader may choose to restrict himself and start with $\mathbf{V}$ satisfying: $\mathrm{GCH}, \lambda=\aleph_{\omega}, \delta(*)=\omega, \lambda_{n}=\aleph_{n(*)+n}, \kappa=\aleph_{n(*)}>\aleph_{1}$ and $\chi=\aleph_{\omega+1}$. Now add $\aleph_{\omega+1}$ generic subsets of $\kappa$, i.e., force with a product of $\chi$ copies of $(\kappa>2, \triangleleft)$ with support $<\kappa$. This model satisfies the hypothesis.

We intend to define a forcing $P$ such that

$$
\mathbf{V}^{P} \models 2^{\aleph_{0}}=\chi+\operatorname{cov}(\text { null })=\lambda+\mathrm{MA}_{<\kappa} .
$$

Definition 2.2. (1) $\mathcal{K}$ is the family of sequences

$$
\bar{Q}=\left(P_{\alpha}, \underline{Q}_{\alpha}, A_{\alpha}, \mu_{\alpha}, \tau_{\alpha}: \alpha<\alpha^{*}\right)
$$

satisfying:

(A) $\left(P_{\alpha}, Q_{\alpha}: \alpha<\alpha^{*}\right)$ is a finite support iteration of c.c.c. forcing notions, we set $\alpha^{*}=\lg (\bar{Q})$ (the length of $\bar{Q}$ ), $P_{\alpha^{*}}$ is the limit.

(B) $\tau_{\alpha} \subseteq \mu_{\alpha}<\kappa$ is the generic of $\underline{Q}_{\alpha}$ (i.e. over $\mathbf{V}^{P_{\alpha}}$ from $G_{Q_{\alpha}}$ we can compute $\tau_{\alpha}$ and vice versa).

(C) $A_{\alpha} \subseteq \alpha$ (for proving Theorem 0.1 we use $\left|A_{\alpha}\right|<\lambda$ ).

(D) $Q_{\alpha}$ is a $P_{\alpha}$-name of a c.c.c. forcing notion but computable from $\left\langle\tau_{\gamma}\left[G_{P_{\alpha}}\right]: \gamma \in A_{\alpha}\right\rangle$; in particular it belongs to $\mathbf{V}_{\alpha}=\mathbf{V}\left[\left\langle\tau_{\gamma}\left[G_{P_{\alpha}}\right]: \gamma \in A_{\alpha}\right\rangle\right]$.

(E) $\alpha^{*} \geq \lambda$ and for $\alpha<\lambda$ we have $Q_{\alpha}=(\omega>2, \triangleleft)$ (the Cohen forcing) and $\mu_{\alpha}=\aleph_{0}$ (well, identifies ${ }^{\omega>} 2$ with $\omega$ ).

(F) For each $\alpha<\alpha^{*}$ one of the following holds (and the case is determined in $\mathbf{V}$, not just a $P_{\alpha}$-name):

( $\alpha)\left|Q_{\alpha}\right|<\kappa,\left|A_{\alpha}\right|<\kappa$ and (just for notational simplicity) the set of elements of $Q_{\alpha}$ is $\mu_{\alpha}<\kappa$ (but the order not necessarily the order of the ordinals) and $Q_{\alpha}$ is separative (i.e. $\zeta \Vdash \xi \in G_{Q_{\alpha}} \Leftrightarrow$ $\left.\underline{Q}_{\alpha} \models \xi \leq \zeta\right)$.

In this case let $\tau_{\alpha}=G_{Q_{\alpha}}$. O.K.

$\left.{ }^{1}\right)$ Actually, any ordinal $\alpha^{*}$ of cardinality $\chi$, divisible by $\chi$ and of cofinality $>\lambda$ is 
( $\beta)$ Essentially $\underline{Q}_{\alpha}=$ Random $^{\mathbf{V}_{\alpha}}\left(=\left\{r \in \mathbf{V}_{\alpha}: r \subseteq^{\omega>} 2\right.\right.$, perfect tree, $\operatorname{Leb}(\lim (r))>0\})$ and $\left|A_{\alpha}\right| \geq \kappa$; but for simplicity $\underline{Q}_{\alpha}=$ Random $^{A_{\alpha}, \bar{Q}\lceil\alpha}$ where for $A \subseteq \lg (\bar{Q})$,

$\operatorname{Random}^{A, \bar{Q}}=\left\{p\right.$ : there is (in $\mathbf{V}$ ) a Borel function $\mathcal{B}=\mathcal{B}\left(x_{0}, x_{1}, \ldots\right)$, with variables ranging on $\{$ true, false $\}$ and range perfect subtrees $r$ of ${ }^{\omega>} 2$ with $\operatorname{Leb}(\lim (r))>0$ such that $(\forall \eta \in r)\left[\operatorname{Leb}\left(\lim \left(r^{[\eta]}\right)\right)>0\right]\left(\right.$ recall $r^{[\eta]}=\{\nu \in r:$ $\nu \unlhd \eta \vee \eta \unlhd \nu\})$, and there are pairs $\left(\gamma_{l}, \zeta_{l}\right)$ for $l<\omega$, with $\gamma_{l} \in A$ and $\zeta_{l}<\mu_{\gamma_{l}}$, such that $p=\mathcal{B}(\ldots$, truth value $\left.\left.\left(\zeta_{l} \in{\underset{\sim}{\gamma_{l}}}_{l}\right), \ldots\right)_{l<\omega}\right\}$

(in other notation, $p=\mathcal{B}$ (truth value $\left.\left(\zeta_{l} \in \tau_{\sim}^{\tau_{l}}\right): l<\omega\right)$ ) and then we let $\operatorname{supp}(p)=\left\{\gamma_{l}: l<\omega\right\}$. In this case $\mu_{\alpha}=\omega$ and $\tau_{\alpha}$ is the random real, i.e.

$$
\tau_{\alpha}(n)=l \Leftrightarrow\left(\exists \eta \in{ }^{n} 2\right)\left[(\omega>2)^{[\eta \frown\langle l\rangle]} \in G_{Q_{\alpha}}\right] .
$$

(2) Let

$P_{\alpha}^{\prime}=\left\{p \in P_{\alpha}\right.$ : for every $\gamma \in \operatorname{dom}(p)$, if $\left|A_{\gamma}\right|<\kappa$ then $p(\gamma)$ is an ordinal $<\mu_{\gamma}$ (not just a $P_{\gamma}$-name) and if $\left|A_{\gamma}\right| \geq \kappa$ then $p(\gamma)$ has the form mentioned in clause $(\mathrm{F})(\beta)$ above (and not just a $P_{\gamma}$-name of such an object) $\}$

(this is a dense subset of $P_{\alpha}$ ).

(3) For $A \subseteq \alpha$ let

$$
P_{A}^{\prime}=\left\{p \in P_{\alpha}^{\prime}: \operatorname{dom}(p) \subseteq A \text { and } \gamma \in \operatorname{dom}(p) \Rightarrow \operatorname{supp}(p(\gamma)) \subseteq A\right\} .
$$

FACT 2.3. Suppose $\bar{Q} \in \mathcal{K}$ with $\lg (\bar{Q})=\alpha^{*}$.

(1) For $\alpha \leq \alpha^{*}, P_{\alpha}^{\prime}$ is a dense subset of $P_{\alpha}$ and $P_{\alpha}$ satisfies the c.c.c.

(2) Suppose

(a) $\operatorname{cf}\left(\alpha^{*}\right)>\lambda$,

(b) for every $A \subseteq \alpha^{*}$, if $|A|<\lambda$, then there is $\beta<\alpha^{*}$ such that $A \subseteq A_{\beta}\left(\right.$ and $\left.\left|A_{\beta}\right| \geq \kappa\right)$.

Then, in the extension, ${ }^{\omega} 2$ is not the union of $<\lambda$ null sets.

(3) In $\mathbf{V}^{P_{\alpha}}$, from $\tau_{\sim \alpha}\left[G_{Q_{\alpha}}\right]$ we can reconstruct $G_{Q_{\alpha}}$ and vice versa. From $\left\langle\tau_{\gamma}: \gamma\langle\alpha\rangle\left[G_{P_{\alpha}}\right]\right.$ we can reconstruct $G_{P_{\alpha}}$ and vice versa. So $\mathbf{V}^{P_{\alpha}}=\mathbf{V}\left[\left\langle\tau_{\beta}\right.\right.$ : $\beta<\alpha\rangle]$.

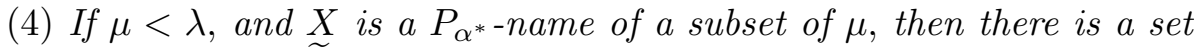
$A \subseteq \alpha^{*}$ such that $|A| \underset{\sim}{\leq} \mu$ and $\Vdash_{P_{\alpha^{*}}} \quad \underset{\sim}{X} \in \mathbf{V}\left[\left\langle\tau_{\sim}: \gamma \in A\right\rangle\right]$ ". Moreover for each $\zeta<\mu$ there is in $\mathbf{V}$ a Borel function $\mathcal{B}\left(x_{0}, \ldots, x_{n}, \ldots\right)_{n<\omega}$ with domain and range the set $\{$ true, false $\}$ and $\gamma_{l} \in A, \zeta_{l}<\mu_{\gamma_{l}}$ for $l<\omega$ such that

$\Vdash_{P_{\alpha^{*}}} " \zeta \in \underset{\sim}{X}$ iff true $=\mathcal{B}\left(\ldots, \text { "truth value of } \zeta_{l} \in \tau_{\gamma_{l}}\left[G_{Q_{\gamma_{l}}}\right] ", \ldots\right)_{l<\omega}$ ". 
(5) For $\bar{Q} \in \mathcal{K}$ and $A \subseteq \alpha^{*}$, every real in $\mathbf{V}\left[\left\langle\tau_{\gamma}: \gamma \in A\right\rangle\right]$ has the form $\mathcal{B}\left(\ldots, \text { truth value }\left(\zeta_{l} \in \tau_{\gamma_{l}}\right), \ldots\right)_{l<\omega}$ where $\gamma_{l}<\alpha^{*}, \zeta_{l}<\mu_{\gamma_{l}}$ and $\mathcal{B}$ is a Borel function (from $\mathbf{V}$ ) from ${ }^{\omega}$ \{true, false $\}$ to ${ }^{\omega} 2$ (the set of reals).

(6) If condition (c) below holds then $\mathbf{V}^{P_{\alpha^{*}}}=\mathrm{MA}_{<\kappa}$, where

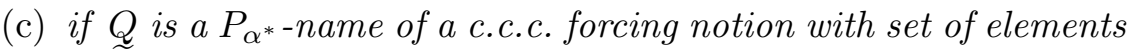
$\mu<\kappa$ then for some $\alpha<\alpha^{*}, \underline{Q}$ is a $P_{\alpha}$-name $\mu_{\alpha}=\mu$ and $\Vdash_{P_{\alpha}} " Q=Q_{\alpha} "$.

(7) If $\left|A_{\beta}\right| \geq \kappa$ and $P_{A_{\beta}}^{\prime}<P_{\beta}$ then $Q_{\beta}$ is actually Random $\mathbf{v}^{P_{A_{\beta}}}$.

(8) If $\left|A_{\alpha}\right|<\lambda$ then $\Vdash_{P_{\alpha}}$ " $Q_{\alpha}<\lambda$ ", in fact $\left|\left\{p(\alpha): p \in P_{\alpha^{*}}^{\prime}\right\}\right|<\lambda$.

Proof. (1) Easy, by induction on $\alpha$.

(2) Easy using parts (3)-(7). Note that for any $\beta<\alpha^{*}$ satisfying $\left|A_{\beta}\right| \geq$ $\kappa$ the null sets from $\mathbf{V}^{\left[\left\langle\tau_{\gamma}: \gamma \in A_{\beta}\right\rangle\right]}$ do not cover ${ }^{\omega} 2$ in $\mathbf{V}^{P_{\alpha^{*}}}$ as we have random reals over $\mathbf{V}^{\left[\left\langle\tau_{\gamma}: \gamma \in A_{\beta}\right\rangle\right]}$. So, by clause (b) of the assumption, it is enough to note that if $\underset{\sim}{y}$ is a $P_{\alpha^{*}}$-name of a member of ${ }^{\omega} 2$, then there is a countable $A \subseteq \alpha^{*}$ such that $\underset{\widetilde{o}}{[}[G] \in \mathbf{V}\left[\left\langle\tau_{\sim}: \beta \in A\right\rangle\right]$. This follows by part (4).

(3) By induction on $\alpha$.

(4) Let $\chi^{*}$ be such that $\{\bar{Q}, \lambda\} \in \mathcal{H}\left(\chi^{*}\right)$, and let $\zeta<\mu$; let $M$ be a countable elementary submodel of $\left(\mathcal{H}\left(\chi^{*}\right), \in,<_{\chi^{*}}^{*}\right)$ to which $\{\bar{Q}, \lambda, \kappa, \mu, \underset{\sim}{X}, \zeta\}$ belongs, so $\Vdash_{P_{\alpha^{*}}}$ " $M\left[G_{P_{\alpha^{*}}}\right] \cap \mathcal{H}\left(\chi^{*}\right)=M^{\prime}$ ". Hence by $2.3(3)$ (i.e. as $\mathbf{V}^{P_{\alpha}}=$ $\left.\mathbf{V}\left[\left\langle\tau_{\beta}: \beta<\alpha\right\rangle\right]\right)$ we have $M\left[G_{P_{\alpha^{*}}}\right]=M\left[\left\langle\tau_{\sim}: i \in \alpha^{*} \cap M\right\rangle\right]$ and the conclusion should be clear.

(5) By 2.3(4).

(6) Straight.

(7) Easy (see more [Sh 619, §3]).

(8) By the definitions (and since $\left(\lambda_{\zeta}\right)^{\aleph_{0}}<\lambda$ and $\lambda=\sup \left\{\lambda_{\zeta}: \zeta<\delta(*)\right\}$ by 2.1 ).

Definition 2.4. (1) Suppose that $\bar{a}=\left\langle a_{l}: l<\omega\right\rangle$ and $\left\langle n_{l}: l<\omega\right\rangle$ are such that:
(a) $a_{l} \subseteq{ }^{n_{l}} 2$
(b) $n_{l}<n_{l+1}<\omega$ for $l<\omega$,
(c) $\left|a_{l}\right| / 2^{n_{l}}>1-1 / 10^{l}$.

Let $N[\bar{a}]=:\left\{\eta \in \omega^{\omega}:\left(\exists^{\infty} l\right)\left(\forall \nu \in a_{l}\right)(\nu \not \eta)\right\}$.

(2) For $\bar{a}$ as above and $n \in \omega$, let

$$
\operatorname{tree}_{n}(\bar{a})=\left\{\nu \in{ }^{\omega>} 2: n_{l}>n \Rightarrow \nu\left\lceil n_{l} \in a_{l}\right\} .\right.
$$

It is well known that for $\bar{a}$ as above the set $N[\bar{a}]$ is null (and $N[\bar{a}]=$ $\left.\omega_{2} \backslash \bigcup_{n<\omega} \lim \left(\operatorname{tree}_{n}(\bar{a})\right)\right)$.

Definition 2.5. For $\alpha<\lambda$ we identify $Q_{\alpha}$ (the Cohen forcing) with $\left\{\left\langle\left(n_{l}, a_{l}\right): l<k\right\rangle: k<\omega, n_{l}<n_{l+1}<\omega, a_{l} \subseteq{ }^{n_{l}} 2,\left|a_{l}\right| / 2^{n_{l}}>1-1 / 10^{l}\right\}$, 
ordered by end extension. If $G_{Q_{\alpha}}$ is $Q_{\alpha}$-generic, let $\left\langle\left(\underline{a}_{l}^{\alpha},{\underset{\sim}{n}}_{l}^{\alpha}\right): l<\omega\right\rangle\left[G_{Q_{\alpha}}\right]$ be the $\omega$-sequence such that every $p \in G_{Q_{\alpha}}$ is an initial segment of it. So we have defined the $Q_{\alpha}$-name $\underline{a}^{\alpha}=\left\langle\underline{a}_{l}^{\alpha}: l<\omega\right\rangle$ and similarly $\left\langle\underline{n}_{l}^{\alpha}: l<\omega\right\rangle$. Let ${\underset{\sim}{\alpha}}_{\alpha}=N\left[\overline{\bar{a}}^{\alpha}\right]$.

Our aim is to prove $(*)_{\bar{Q}}$, where

Definition 2.6. For $\bar{Q} \in \mathcal{K}$ with $\alpha^{*}=\lg (\bar{Q})$ let $(*)_{\bar{Q}} \quad\left\langle N_{\alpha}: \alpha<\lambda\right\rangle$ cover ${ }^{\omega} 2$ in $\mathbf{V}^{P_{\alpha^{*}}}$, where $P_{\alpha^{*}}=\operatorname{Lim}(\bar{Q})$.

We shall eventually prove it not for every $\bar{Q}$, but for enough $\bar{Q}$ 's (basically asking the $A_{\alpha}$ of cardinality $\geq \kappa$ to be closed enough).

Lemma 2.7. For $\bar{Q} \in \mathcal{K}$ with $\gamma=\lg (\bar{Q})$, a sufficient condition for $(*)_{\bar{Q}}$ is:

$(* *)_{\bar{Q}} \quad$ In $\mathbf{V}^{P_{\gamma}}$, there is no perfect tree $T \subseteq{ }^{\omega>} 2$ and $E \in[\lambda]^{\kappa^{+}}$such that, for some $n<\omega, T \subseteq \operatorname{tree}_{n}\left[\bar{a}^{\alpha}\right]$ for all $\alpha \in E$.

Proof. By induction on $\gamma \geq \lambda$. For $\gamma=\lambda$, trivial by properties of the Cohen forcing.

Suppose $\gamma>\lambda$ is limit. Assume toward contradiction that

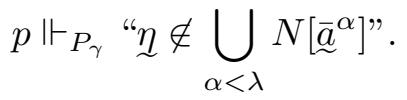

Without loss of generality,

$$
p \Vdash_{P_{\gamma}} " \eta \notin \mathbf{V}^{P_{\beta}} "
$$

for every $\beta<\gamma$, hence by properties of FS iteration of c.c.c. forcing notions, $\operatorname{cf}(\gamma)=\aleph_{0}$. So for each $\alpha<\lambda$ there are $p_{\alpha}, m_{\alpha}$ such that

$$
p \leq p_{\alpha} \in P_{\gamma}, \quad p_{\alpha} \Vdash “ \eta \in \lim \left(\operatorname{tree}_{m_{\alpha}}\left(\bar{a}^{\alpha}\right)\right) " .
$$

Note that (by properties of c.c.c. forcing notions) $\left\langle\left\{\alpha<\lambda: p_{\alpha} \in P_{\beta}\right\}\right.$ : $\beta<\gamma\rangle$ is an increasing sequence of subsets of $\lambda$ of length $\gamma$, so for some $\gamma_{1}<\gamma$ there is $E \in[\lambda]^{\kappa^{+}}$such that $p_{\alpha} \in P_{\gamma_{1}}$ for every $\alpha \in E$ and we can assume $m_{\alpha}=m$ for $\alpha \in E$. Note that for all but $<\kappa^{+}$of the ordinals $\alpha \in E$ we have

$$
p_{\alpha} \Vdash "\left|\left\{\beta \in E: p_{\beta} \in G_{P_{\gamma_{1}}}\right\}\right|=\kappa^{+"} .
$$

Fix such an $\alpha$, and let $G_{P_{\gamma_{1}}}$ be a $P_{\gamma_{1}}$-generic (over $\mathbf{V}$ ) subset of $P_{\gamma_{1}}$ to which $p_{\alpha}$ belongs. Now in $\mathbf{V}\left[G_{P_{\gamma_{1}}}\right]$ let $E^{\prime}=\left\{\beta \in E: p_{\beta} \in G_{P_{\gamma_{1}}}\right\}$ so $\left|E^{\prime}\right|=\kappa^{+}$. Let $T^{*}=\bigcap_{\beta \in E^{\prime}}$ tree $_{m}\left(\bar{a}^{\beta}\right)$. Note that, in $\mathbf{V}^{P_{\gamma_{1}}}, T^{*}$ is a subtree of $\omega>_{2}$ and by $(* *)_{\bar{Q}}, T^{*}$ contains no perfect subtree. Hence $\lim \left(T^{*}\right)$ is countable, so absolute. But $p_{\alpha} \Vdash_{P_{\gamma}} " \eta \in \lim \left({\underset{\sim}{T}}^{*}\right)$ ", so $p_{\alpha} \Vdash " \eta \underline{\mathbf{V}^{P_{\gamma_{1}}}}$ ", a contradiction.

Assume now that $\gamma=\beta+1>\lambda$ and work in $\mathbf{V}^{P_{\beta}}$. Choose $p, p_{\alpha} \in Q_{\beta}$ as before. By 2.3(7), $Q_{\beta}$ has a dense subset of cardinality $<\lambda$, so there are 
$q \in Q_{\beta}$ and $m$ such that $E=\left\{\alpha<\lambda: m_{\alpha}=n, p_{\alpha} \leq q\right\}$ has cardinality $\geq \kappa^{+}$. Continue as above.

As we have covered the cases $\gamma=\lambda, \gamma>\lambda$ limit and $\gamma>\lambda$ successor, we have finished the proof.

Discussion 2.8. Note that by Lemma 2.7 and Fact 2.3 it is enough to show that there is $\bar{Q} \in \mathcal{K}$ such that $\alpha^{*}=\lg (\bar{Q})$ (where $\alpha^{*}$ is chosen as the length of the final iteration from 2.1(b)), satisfying clauses (a)+(b)+(c) of Fact $2.3(2)+(6)$ and $(* *)_{\bar{Q}}$. To prove the latter we need to impose more restrictions on the iteration. Now when Haim Judah asked me on the problem, whereas 2.1-2.7 were quite immediate, arriving at 2.9-2.11 has taken me many years and much effort.

Definition 2.9. $\mathcal{T}$, the set of blueprints, is the set of tuples

$$
t=\left(w^{t}, \mathbf{n}^{t}, \mathbf{m}^{t}, \bar{\eta}^{t}, h_{0}^{t}, h_{1}^{t}, h_{2}^{t}, \bar{n}^{t}\right)
$$

where:

(a) $w^{t} \in[\kappa]^{\aleph_{0}}$

(b) $0<\mathbf{n}^{t}<\omega, \mathbf{m}^{t} \leq \mathbf{n}^{t}$,

(c) $\bar{\eta}^{t}=\left\langle\eta_{\mathbf{n}, k}^{t}: \mathbf{n}<\mathbf{n}^{t}, k<\omega\right\rangle, \eta_{\mathbf{n}, k}^{t} \in w^{t} 2$ for $\mathbf{n}<\mathbf{n}^{t}, k<\omega$,

(d) $h_{0}^{t}$ is a partial function from $\left[0, \mathbf{n}^{t}\right)$ to ${ }^{\omega} \kappa$, its domain includes the set $\left\{0, \ldots, \mathbf{m}^{t}-1\right\}$ (here we consider members of $Q_{\alpha}$ for $\alpha<\lambda$ as integers $\left({ }^{2}\right)$ ),

(e) $h_{1}^{t}$ is a partial function from $\left[0, \mathbf{n}^{t}\right)$ to $(0,1)_{\mathbb{Q}}$ (rationals), but for $\mathbf{n} \in$ $\left[0, \mathbf{n}^{t}\right) \backslash \operatorname{dom}\left(h_{1}^{t}\right)$ we stipulate $h_{1}^{t}(\mathbf{n})=0$ and we assume $\sum_{\mathbf{n}<\mathbf{n}^{t}} \sqrt{h_{1}^{t}(\mathbf{n})}<$ $1 / 10$.

(f) $h_{2}^{t}$ is a partial function from $\left[0, \mathbf{n}^{t}\right)$ to ${ }^{\omega>} 2$,

(g) $\operatorname{dom}\left(h_{0}^{t}\right), \operatorname{dom}\left(h_{1}^{t}\right)$ are disjoint with union $\left[0, \mathbf{n}^{t}\right)$,

(h) $\operatorname{dom}\left(h_{2}^{t}\right)=\operatorname{dom}\left(h_{1}^{t}\right)$,

(i) $\eta_{\mathbf{n}_{1}, k_{1}}^{t}=\eta_{\mathbf{n}_{2}, k_{2}}^{t} \Rightarrow \mathbf{n}_{1}=\mathbf{n}_{2}$,

(j) for each $\mathbf{n}<\mathbf{n}^{t}$ we have: $\left\langle\eta_{\mathbf{n}, k}^{t}: k<\omega\right\rangle$ is constant or with no repetitions; if it is constant and $\mathbf{n} \in \operatorname{dom}\left(h_{0}^{t}\right)$ then $h_{0}^{t}(\mathbf{n})$ is constant,

(k) $\bar{n}^{t}=\left\langle n_{k}^{t}: k<\omega\right\rangle$ where $n_{0}^{t}=0, n_{k}^{t}<n_{k+1}^{t}<\omega$ and the sequence $\left\langle n_{k+1}^{t}-n_{k}^{t}: k<\omega\right\rangle$ goes to infinity. For $l<\omega$ and such $\bar{n}$ let $k_{\bar{n}}(l)=k(l, \bar{n})$ be the unique $k$ such that $n_{k} \leq l<n_{k+1}$.

Discussion 2.10. The definitions of a blueprint $t \in \mathcal{T}$ (in Definition 2.9) and of iterations $\bar{Q} \in \mathcal{K}^{3}$ (defined in Definition 2.11(c) below; the reader may first read it) contain the main idea of the proof, so though they have many clauses, the reader is advised to try to understand them.

$\left({ }^{2}\right)$ Actually the case where each $h_{0}^{t}(\mathbf{n})$ is a constant function from $\omega$ to $\kappa$ suffices, and so $\kappa<\lambda$ suffices instead of $\kappa^{\aleph_{0}}<\lambda$. 
In order to prove $(* *)_{\bar{Q}}$ we will show in $\mathbf{V}^{P_{\alpha *}}$ that if $E \in[\lambda]^{\kappa^{+}}$and $n<\omega$, then $\bigcap_{\alpha \in E} \operatorname{tree}_{n}\left(\overline{\bar{a}}^{\alpha}\right)$ is a tree with finitely many branches. So let $p$ be given, let $p \leq p_{\zeta} \Vdash$ " $\beta_{\zeta} \in \underset{\sim}{E}$ " for $\zeta<\kappa^{+}, \beta_{\zeta} \notin\left\{\beta_{\xi}: \xi<\zeta\right\}$, we can assume $p_{\zeta}$ is in some pregiven dense set, and $\left\langle p_{\zeta}: \zeta<\kappa^{+}\right\rangle$form a $\Delta$-system (with some more "thinning" demands), $\operatorname{dom}\left(p_{\zeta}\right)=\left\{\alpha_{\mathbf{n}, \zeta}: \mathbf{n}<\mathbf{n}^{*}\right\}, \alpha_{\mathbf{n}, \zeta}$ is increasing with $n$, and $\alpha_{\mathbf{n}, \zeta}<\lambda$ iff $\mathbf{n}<\mathbf{m}^{*}$. Let $p_{\zeta}^{\prime}$ be $p_{\zeta}$ when $p_{\zeta}\left(\alpha_{\mathbf{n}, \zeta}\right)$ is increased a little, as described below.

It suffices to find $p^{*} \geq p$ such that $p^{*} \Vdash$ "A $\underset{\sim}{A}:=\left\{\zeta<\omega: p_{\zeta}^{\prime} \in G\right\}$ is large enough such that $\bigcap_{\zeta \in \mathcal{\sim}_{\text {促 }}} \operatorname{tree}_{m}\left(\bar{a}^{\beta_{\zeta}}\right)$ has only finitely many branches".

Because of "communication problems" the "large enough" is interpreted as of $\Xi_{\alpha}^{t}$-measure (again defined in 2.11 below).

The natural numbers $\mathbf{n}<\mathbf{n}^{*}$ such that $Q_{\alpha_{\mathbf{n}, \zeta}}$ is a forcing notion of cardinality $<\kappa$ do not cause problems, as $h_{0}^{t}(\mathbf{n})$ tells us exactly what the condition $p_{\zeta}\left(\alpha_{\mathbf{n}, \zeta}\right)$ is. Still there are many cases of such $\left\langle p_{\zeta}: \zeta<\omega\right\rangle$ which fall into the same $t$; we possibly will get contradictory demands if $\alpha_{\mathbf{n}_{1}, \zeta_{1}}=$ $\alpha_{\mathbf{n}_{2}, \zeta_{2}}, \mathbf{n}_{1} \neq \mathbf{n}_{2}$. But the $w^{t}, \bar{\eta}^{t}$ are exactly built to make this case not to happen. That is, we have to assume $2^{\kappa}=\chi\left(=\left|\alpha^{*}\right|\right)$ in order to be able for our iteration $\left\langle P_{\alpha}, Q_{\alpha}: \alpha<\alpha^{*}\right\rangle$ to choose $\left\langle\eta_{\alpha}: \alpha<\alpha^{*}\right\rangle, \eta_{\alpha} \in{ }^{\kappa} 2$, with no repetitions, so that if $v \subseteq \chi,|v| \leq \aleph_{0}$ (e.g. $\left.v=\left\{\alpha_{\mathbf{n}, \zeta}: \mathbf{n}<\mathbf{n}^{t}, \zeta<\omega\right\}\right)$ then for some $w=w^{t} \in[\kappa]^{\aleph_{0}}$ we have $\left\langle\eta_{\alpha}\lceil w: \alpha \in v\rangle\right.$ with no repetitions.

So the blueprint $t$ describes such a situation, giving as much information as possible, as long as the number of blueprints is not too large, $\kappa^{\aleph_{0}}=\kappa$ in our case.

If $Q_{\alpha_{\mathbf{n}, \zeta}}$ is a partial random, we may get many candidates for $p_{\zeta}\left(\alpha_{\mathbf{n}, \zeta}\right) \in$ Random and they are not all the same ones. We want them in many cases to be in the generic set. Well, we can (using $\left.h_{1}^{t}(\mathbf{n}), h_{2}^{t}(\mathbf{n})\right)$ know that in some interval $\left({ }^{\omega} 2\right)^{\left[h_{2}^{t}(\mathbf{n})\right]}$ the set $\lim \left(p_{\zeta}\left(\alpha_{\mathbf{n}, \zeta}\right)\right)$ is large, say of relative measure $\geq 1-h_{1}^{t}(\mathbf{n})$, and we could have chosen the $p_{\zeta}$ 's such that $\left\langle h_{1}^{t}(\mathbf{n}): \mathbf{n}<\mathbf{n}^{t}\right\rangle$ is small enough, still the number of candidates is not bounded by $1 / h_{1}^{t}(\mathbf{n})$. Here taking limit along ultrafilters is not good enough, but using finitely additive measures is.

Well, we have explained $w^{t}, \bar{\eta}^{t}, h_{0}^{t}, h_{1}^{t}, h_{2}^{t}$, but what about the $\bar{n}^{t}=$ $\left\langle n_{k}^{t}: k<\omega\right\rangle$ ? In the end (in $\S 3$ ) the specific demand on $\left\{\zeta: p_{\zeta}^{\prime} \in G\right\}$ being large, is that for infinitely many $k<\omega$,

$$
\mid\left\{l: n_{k}^{t} \leq l<n_{k+1}^{t} \text { and } p_{l} \in G\right\} \mid /\left(n_{k+1}^{t}-n_{k}^{t}\right)
$$

is large, the $n_{k}^{t}$ will be chosen such that it is increasing fast enough and $\left\langle p_{l}^{\prime}\left(\beta_{l}\right): l \in\left[n_{k}^{t}, n_{k+1}^{t}\right)\right\rangle$ will be chosen such that for each $\varepsilon>0$ for some $s<\omega$, for $k$ large enough if the above fraction is $\geq \varepsilon$ then essentially ${ }^{k} 2 \cap\left\{\operatorname{tree}_{m}\left(\bar{a}^{\beta_{l}}\right): n_{k}^{t} \leq l<n_{k+1}^{t}\right.$ and $\left.p_{l}^{\prime} \in G\right\}$ has $\leq s$ members; this suffices. 
What is our plan? We define $\mathcal{K}^{3}$, the class of suitable expanded iterations $\bar{Q}$ by choice of $\eta_{\alpha}$ (for $\alpha<\lg (\bar{Q})$ ) and names for finitely additive measures $\Xi_{\alpha}^{t}$ satisfying the demands natural in this context. You may wonder why we use $\Xi$-averages; this is like integral or expected value, and so they "behave nicely" making the "probability computations" simpler. Then we show that we can find $\bar{Q} \in \mathcal{K}^{3}$ in which all obligations toward "cov(null) $\geq \lambda$ " and $\mathrm{MA}_{<\kappa}$ hold.

The main point of $\S 3$ will be that we can carry out the argument of "for some $p^{*}$ we have $p^{*} \Vdash\left\{l<\omega: p_{l}^{\prime} \in G\right\}$ is large" and why it gives $n<\omega \& E \in[\chi]^{\kappa^{+}} \Rightarrow \bigcap_{\zeta \in A} \operatorname{tree}_{n}\left(\bar{a}^{\zeta}\right)$ has finitely many branches, thus proving Theorem 0.1 .

The reader may wonder how much the $\underset{\sim}{\underset{\sim}{\Xi}}{ }_{\alpha}^{t}$ are actually needed. As explained above they are just a transparent way to express the property; this will be utilized in [Sh 619].

Definition 2.11. $\quad \mathcal{K}^{3}$ is the class of sequences

$$
\bar{Q}=\left\langle P_{\alpha}, Q_{\beta}, A_{\beta}, \mu_{\beta}, \tau_{\beta}, \eta_{\beta},\left(\Xi_{\sim}^{t}\right)_{t \in \mathcal{T}}: \alpha \leq \alpha^{*}, \beta<\alpha^{*}\right\rangle
$$

(we write $\alpha^{*}=\lg (\bar{Q})$ ) such that:

(a) $\left\langle P_{\alpha}, \underline{Q}_{\beta}, A_{\beta}, \mu_{\beta}, \tau_{\beta}: \alpha \leq \alpha^{*}, \beta<\alpha^{*}\right\rangle$ is in $\mathcal{K}$,

(b) $\eta_{\beta} \in{ }^{\kappa} 2$ and $\left(\forall \beta<\alpha<\alpha^{*}\right)\left[\eta_{\alpha} \neq \eta_{\beta}\right]$,

(c) $\mathcal{T}$ is from Definition 2.9, and $\underset{\sim}{\Xi_{\alpha}^{t}}$ is a $P_{\alpha}$-name of a finitely additive measure on $\omega$ (in $\mathbf{V}^{P_{\alpha}}$, i.e. $\left.\in\left(\mathcal{M}^{\text {full }}\right)^{\mathbf{V}^{P_{\alpha}}}\right)$, increasing with $\alpha$,

(d) we say that $\bar{\alpha}=\left\langle\alpha_{l}: l<\omega\right\rangle$ satisfies $(t, \mathbf{n})$ (for $\bar{Q}$ ) if:

- $\left\langle\alpha_{l}: l<\omega\right\rangle \in \mathbf{V}$ (of course),

- $t \in \mathcal{T}, \mathbf{n}<\mathbf{n}^{t}$,

- $\alpha_{l} \leq \alpha_{l+1}<\alpha^{*}$

- $\mathbf{n}<\mathbf{m}^{t} \Leftrightarrow(\forall l)\left(\alpha_{l}<\lambda\right) \Leftrightarrow(\exists k)\left(\alpha_{k}<\lambda\right)$,

- $\eta_{\mathbf{n}, l}^{t} \subseteq \eta_{\alpha_{l}}$ (as functions),

- if $\mathbf{n} \in \operatorname{dom}\left(h_{0}^{t}\right)$, then $\mu_{\alpha_{l}}<\kappa$ and $\Vdash_{P_{\alpha_{l}}} "\left|Q_{\alpha_{l}}\right|<\kappa$ and $\left(h_{0}^{t}(\mathbf{n})\right)(l) \in \underline{Q}_{\alpha_{l}}$, i.e. $\left(h_{0}^{t}(\mathbf{n})\right)(l)<\mu_{\alpha_{l}} "$,

- if $\mathbf{n} \in \operatorname{dom}\left(h_{1}^{t}\right)$, then $\mu_{\alpha_{l}} \geq \kappa$ so $\Vdash_{P_{\alpha_{l}}}$ " $Q_{\alpha_{l}}$ has cardinality $\geq \kappa$ " (so it is a partial random),

- if $\left\langle\eta_{\mathbf{n}, k}^{t}: k \in \omega\right\rangle$ is constant, then $(\forall l)\left[\alpha_{l}=\alpha_{0}\right]$; if $\left\langle\eta_{\mathbf{n}, k}^{t}: k<\omega\right\rangle$ is not constant, then $(\forall l)\left[\alpha_{l}<\alpha_{l+1}\right]$,

(e) if $\bar{\alpha}=\left\langle\alpha_{l}: l<\omega\right\rangle$ satisfies $(t, \mathbf{n})$ for $\bar{Q}, \bigwedge_{l<\omega}\left(\alpha_{l}<\alpha_{l+1}\right), \mathbf{n} \in$ $\operatorname{dom}\left(h_{0}^{t}\right)$ then

$\Vdash_{P_{\alpha^{*}}}$ "the following set has $\underset{\sim}{\Xi_{\alpha^{*}}} t$-measure 1:

$$
\left\{k<\omega: \text { if } l \in\left[n_{k}^{t}, n_{k+1}^{t}\right) \text { then }\left(h_{0}^{t}(\mathbf{n})\right)(l) \in G_{Q_{\alpha_{l}}}\right\} ",
$$


(f) if $\bar{\alpha}=\left\langle\alpha_{l}: l<\omega\right\rangle$ satisfies $(t, \mathbf{n})$ for $\bar{Q}, \mathbf{n} \in \operatorname{dom}\left(h_{1}^{t}\right),(\forall l<\omega)\left[\alpha_{l}<\right.$ $\left.\alpha_{l+1}\right]$, and $\bar{r}=\left\langle\underline{r}_{l}: l<\omega\right\rangle$ where for $l<\omega, \underline{r}_{l}$ is a $P_{\alpha_{l}}$-name of a member of $Q_{\alpha_{l}}$ such that (it is forced that)

$$
1-h_{1}^{t}(\mathbf{n}) \leq \operatorname{Leb}\left(\left\{\eta \in \omega^{\omega} 2: h_{2}^{t}(\mathbf{n}) \triangleleft \eta \in \lim \left(\underline{r}_{l}\right)\right\}\right) / 2^{\lg \left(h_{2}^{t}(\mathbf{n})\right)},
$$

then for each $\varepsilon>0$ we have

$\Vdash_{P_{\alpha^{*}}}$ "the following set has $\underset{\Xi_{\alpha^{*}}}{t}$-measure 1:

$\left\{k<\omega:\right.$ in the set $\left\{l \in\left[n_{k}^{t}, n_{k+1}^{t}\right): \underline{r}_{l} \in G_{Q_{\alpha_{l}}}\right\}$ there are

$$
\text { at least } \left.\left(n_{k+1}^{t}-n_{k}^{t}\right)\left(1-h_{1}^{t}(\mathbf{n})\right)(1-\varepsilon) \text { elements }\right\} ",
$$

(g) if $\bar{\alpha}=\left\langle\alpha_{l}: l<\omega\right\rangle$ satisfies $(t, \mathbf{n})$ for $\bar{Q}, \mathbf{n} \in \operatorname{dom}\left(h_{1}^{t}\right),(\forall l)\left[\alpha_{l}=\alpha\right]$, and $\underset{\sim}{r}, \underline{r}_{l}$ are $P_{A_{\alpha}}^{\prime}$-names of members of $Q_{\alpha}$ satisfying $(* *)_{\left.\underset{\sim}{Q}, \underline{\sim}_{l}: l<\omega\right\rangle}^{\bar{Q}}$ (see below for the definition of $(* *))$ then

$\Vdash_{P_{\alpha^{*}}}$ "if $\underset{\sim}{r} \in G_{Q_{\alpha}}$ then

$$
1-h_{1}^{t}(\mathbf{n}) \leq \operatorname{Av}_{\Xi_{\alpha^{*}}^{t}}\left(\left\langle\left|\left\{l \in\left[n_{k}^{t}, n_{k+1}^{t}\right): \underline{r}_{l} \in G_{Q_{\alpha}}\right\}\right| /\left(n_{k+1}^{t}-n_{k}^{t}\right): k<\omega\right\rangle\right) ",
$$

where

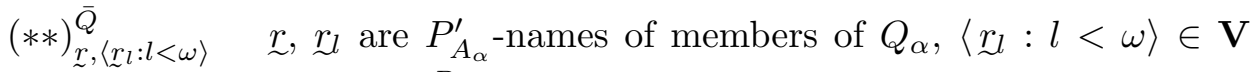
and, in $\mathbf{V}^{P_{\alpha}}$, for every $r^{\prime} \in Q_{\alpha}$ satisfying $r \leq r^{\prime}$ we have

where

$$
\operatorname{Av}_{\Xi_{\alpha}^{t}}\left(\left\langle a_{k}\left(r^{\prime}\right): k<\omega\right\rangle\right) \geq 1-h_{1}^{t}(\mathbf{n})
$$

$$
\begin{aligned}
a_{k}\left(r^{\prime}\right) & =a_{k}\left(r^{\prime}, \bar{r}\right)=a_{k}\left(r^{\prime}, \bar{r}, \bar{n}^{t}\right) \\
& =\left(\sum_{l \in\left[n_{k}^{t}, n_{k+1}^{t}\right)} \frac{\operatorname{Leb}\left(\lim \left(r^{\prime}\right) \cap \lim \left(r_{l}\right)\right)}{\operatorname{Leb}\left(\lim \left(r^{\prime}\right)\right)}\right) \frac{1}{n_{k+1}^{t}-n_{k}^{t}}
\end{aligned}
$$

(so $a_{k}\left(r^{\prime}, \bar{r}, \bar{n}\right) \in[0,1]_{\mathbb{R}}$ is well defined for $k<\omega, \bar{r}=\left\langle r_{l}: l<\omega\right\rangle,\left\{r, r_{l}\right\} \subseteq$ Random, $\left.\bar{n}=\left\langle n_{l}: l<\omega\right\rangle, n_{l}<n_{l+1}<\omega\right)$,

(h) $\left|A_{\alpha}\right| \geq \kappa \Rightarrow P_{A_{\alpha}}^{\prime}<P_{\alpha}$, and $\left(^{3}\right) \beta \in A_{\alpha} \&\left|A_{\beta}\right|<\kappa \Rightarrow A_{\beta} \subseteq A_{\alpha}$,

(i) if $\vdash_{P_{\alpha}}$ "| $\left|\underline{Q}_{\alpha}\right| \geq \kappa$ ", then $\underset{\sim}{\Xi} \mid \mathcal{P}(\omega)^{\mathbf{V}^{P_{A_{\alpha}}}}$ is a $P_{A_{\alpha}}$-name $\left({ }^{4}\right)$ for every $t \in \mathcal{T}$.

Definition 2.12. (1) For $\bar{Q} \in \mathcal{K}^{3}$ and for $\alpha^{*} \leq \lg (\bar{Q})$ let

$$
\bar{Q}\left\lceil\alpha^{*}=\left\langle P_{\alpha}, Q_{\beta}, A_{\beta}, \mu_{\beta}, \tau_{\beta}, \eta_{\beta},\left(\Xi_{\alpha}^{t}\right)_{t \in \mathcal{T}}: \alpha \leq \alpha^{*}, \beta<\alpha^{*}\right\rangle .\right.
$$

(2) For $\bar{Q}^{1}, \bar{Q}^{2} \in \mathcal{K}^{3}$ we say: $\bar{Q}^{1} \leq \bar{Q}^{2}$ if $\bar{Q}^{1}=\bar{Q}^{2} \uparrow \lg \left(\bar{Q}^{1}\right)$.

FACT 2.13. (1) If $\bar{Q} \in \mathcal{K}^{3}$ and $\alpha \leq \lg (\bar{Q})$, then $\bar{Q}\left\lceil\alpha \in \mathcal{K}^{3}\right.$.

(2) $\left(\mathcal{K}^{3}, \leq\right)$ is a partial order.

$\left({ }^{3}\right)$ If we add " $(\forall \alpha<\kappa)\left(|\alpha|^{\aleph_{0}}<\kappa\right)$ " to 2.1 , we can simplify and assume $\alpha<\alpha^{*} \Rightarrow$ $P_{A_{\alpha}}^{\prime}<P_{\alpha}$

$\left({ }^{4}\right)$ Here the secret was whispered. 
(3) If a sequence $\left\langle\bar{Q}^{\beta}: \beta<\delta\right\rangle \subseteq \mathcal{K}^{3}$ is increasing and $\operatorname{cf}(\delta)>\aleph_{0}$, then there is a unique $\bar{Q} \in \mathcal{K}^{3}$ which is the least upper bound, $\lg (\bar{Q})=$ $\bigcup_{\beta<\delta} \lg \left(\bar{Q}^{\beta}\right)$ and $\bar{Q}^{\beta} \leq \bar{Q}$ for all $\beta<\delta$.

Proof. Easy (recall that it is well known that $\left({ }^{\omega_{2}}\right)^{\mathbf{V}^{P_{\delta}}}=\bigcup_{\beta<\delta}\left({ }^{\omega_{2}}\right)^{\mathbf{V}^{P_{\beta}}}$, so $\underset{\sim}{\Xi}=\bigcup_{\beta<\delta} \Xi_{\beta}^{t}$ is a legal choice; this is the use of $\left.\operatorname{cf}(\delta)>\aleph_{0}\right)$.

Lemma 2.14. Suppose that $\bar{Q}^{n} \in \mathcal{K}^{3}, \bar{Q}^{n}<\bar{Q}^{n+1}, \alpha_{n}=\lg \left(\bar{Q}^{n}\right), \delta=$ $\sup _{n<\omega} \alpha_{n}$. Then there is $\bar{Q} \in \mathcal{K}^{3}$ such that $\lg (\bar{Q})=\delta$ and $\bar{Q}^{n} \leq \bar{Q}$ for $n \in \omega$.

Proof. Note that the only problem is to define $\Xi_{\delta}^{t}$ for $t \in \mathcal{T}$, i.e., we have to extend $\bigcup_{\alpha<\delta} \Xi_{\alpha}^{t}$ so that the following two conditions are satisfied (they correspond to clauses (f) and (e) of Definition 2.11).

(a) We are given $\left(^{5}\right) \mathbf{n}<\mathbf{n}^{t}, \mathbf{n} \in \operatorname{dom}\left(h_{1}^{t}\right),\left\langle\alpha_{l}: l<\omega\right\rangle$ (from $\mathbf{V}$ of course) satisfies $(t, \mathbf{n})$ for $\bar{Q}$ and is strictly increasing with limit $\delta$ and we are given $\left\langle\underset{\sim}{p_{l}}: l<\omega\right\rangle$ such that $\Vdash_{P_{\alpha_{l}}}{ }_{\sim}^{p_{l}} \in{\underset{\sim}{\alpha_{l}}}_{\alpha_{l}}$ and $1-h_{1}^{t}(\mathbf{n}) \leq \operatorname{Leb}(\{\eta \in$ $\left.\left.\omega_{2}: h_{2}^{t}(\mathbf{n}) \triangleleft \eta \in \lim \left(\sim_{l}\right)\right\}\right) / 2^{\lg \left(h_{2}^{t}(\mathbf{n})\right)}$ ". The demand is: for each $\varepsilon>0$ we have $\Vdash_{P_{\delta}}$ " $\Xi_{\delta}^{t}(C)=1$ ", where

$$
\begin{array}{r}
\underset{\sim}{C}=\left\{k<\omega: \text { in the set }\left\{l: l \in\left[n_{k}^{t}, n_{k+1}^{t}\right) \text { and } p_{l} \in G_{Q_{\alpha_{l}}}\right\}\right. \text { there are } \\
\text { at least } \left.\left(n_{k+1}^{t}-n_{k}^{t}\right)\left(1-h_{1}^{t}(\mathbf{n})\right)(1-\varepsilon) \text { elements }\right\} .
\end{array}
$$

(b) If $\left(^{4}\right) \mathbf{n}<\mathbf{n}^{t}, \mathbf{n} \in \operatorname{dom}\left(h_{0}^{t}\right),\left\langle\alpha_{l}: l<\omega\right\rangle$ satisfies $(t, \mathbf{n})$ for $\bar{Q}$ and is strictly increasing with limit $\delta$, and $p_{l} \in Q_{\alpha_{l}}$ satisfy $p_{l}=h_{0}^{t}(\mathbf{n})(l)$ for $l<\omega$ (an ordinal $<\mu_{\alpha_{l}}$ ), then $\vdash_{P_{\delta}}$ “寻 $\sigma_{\delta}^{t}(\underset{\sim}{C)}=1$ " where

$$
\underset{\sim}{C}=\left\{k<\omega: \text { for every } l \in\left[n_{k}, n_{k+1}\right) \text { we have } p_{l} \in G_{Q_{\alpha_{l}}}\right\} .
$$

As $\bigcup_{\alpha<\delta} \Xi_{\alpha}^{t}$ is a $\left(P_{\delta}\right.$-name of a) member of $\mathcal{M}$ in $\mathbf{V}^{P_{\delta}}$, by $1.3(3)$ it suffices to prove

(*) $\quad \Vdash_{P_{\delta}}$ "if $\underset{\sim}{B} \in \bigcup_{\alpha<\delta} \operatorname{dom}\left({\underset{\sim}{\sim}}_{\alpha}^{t}\right)=\bigcup_{\alpha<\delta} \mathcal{P}(\omega) \mathbf{V}\left[P_{\alpha}\right]$ and $\underset{\sim}{\Xi_{\alpha}^{t}}(\underset{\sim}{B})>0$ and $j^{*}<\omega$, and $C_{j}$ (for $j<j^{*}$ ) are from (a), (b) above then $\underset{\sim}{B} \cap \bigcap_{j<j^{*}} \underset{\sim}{C} \neq \emptyset^{\prime \prime}$.

Toward contradiction assume $q \in P_{\delta}$ force the negation, so possibly increasing $q$ we have: for some $\underset{\widetilde{B}}{B}$ and for some $j^{*}<\omega$, for each $j<j^{*}$ we have the $\varepsilon_{j}>0$, and $\left.\mathbf{n}(j)<\mathbf{n}^{t}, \widetilde{\langle\alpha}_{l}^{j}: l<\omega\right\rangle,\left\langle p_{l}^{j}: l<\omega\right\rangle$ involved in the definition of ${\underset{\sim}{j}}_{j}$ (in (a) or (b) above), $q$ force: $\underset{\sim}{B} \in \bigcup_{\alpha<\delta} \operatorname{dom}\left({\underset{\sim}{\alpha}}_{\alpha}^{t}\right)=\bigcup_{\alpha<\delta} \mathcal{P}(\omega)^{\mathbf{V}\left[P_{\alpha}\right]}$ and $\left(\bigcup_{\alpha<\delta}{\underset{\widetilde{\Xi}}{\Xi}}_{\alpha}^{t}\right)(\underset{\sim}{B})>0$ and ${\underset{\sim}{j}}_{j}$ (for $j<j^{*}$ ) comes from (a) or (b) above, but $\underset{\sim}{B} \cap \bigcap_{j<j^{*}} \widetilde{\widetilde{C}}_{j}=\widetilde{\emptyset}$; as we can decrease $\varepsilon$, without loss of generality $\varepsilon_{j}=\varepsilon$. Again we can assume that for some $\alpha(*)<\delta, \underset{\sim}{B} \in \operatorname{dom}\left(\Xi_{\alpha(*)}^{t}\right)$ is a $P_{\alpha(*)^{-}}$ name, and $C_{j}$ have the $\mathbf{n}(j)<\mathbf{n}^{t},\left\langle\alpha_{l}^{j}: l<\omega\right\rangle,\left\langle p_{l}^{j}: l<\omega\right\rangle$ witnessing it is as

$\left(^{5}\right)$ In $\mathbf{V}$, so $\left\langle\left(\alpha_{l}, p_{l}\right): l<\omega\right\rangle \in \mathbf{V}$, of course. 
required in (a) or (b) above. Without loss of generality, $q \in P_{\alpha(*)}$. Possibly increasing $q$ (inside $P_{\alpha(*)}$ though) we can find $k<\omega$ such that $q \vDash " k \in \underset{\sim}{B}$ " and $\bigwedge_{j<j^{*}} \bigwedge_{l \in\left[n_{k}^{t}, n_{k+1}^{t}\right)} \alpha_{l}^{j}>\alpha(*)$ and moreover such that $n_{k+1}^{t}-n_{k}^{t}$ is large enough compared to $1 / \varepsilon, j^{*}$ (just let $q \in G_{P_{\alpha(*)}} \subseteq P_{\alpha(*)}, G_{P_{\alpha(*)}}$ generic over $\mathbf{V}$ and think in $\left.\mathbf{V}\left[G_{P_{\alpha(*)}}\right]\right)$. Let $\left\{\alpha_{l}^{j}: j<j^{*}\right.$ and $\left.l \in\left[n_{k}^{t}, n_{k+1}^{t}\right)\right\}$ be listed as $\left\{\beta_{m}: m<m^{*}\right\}$, in increasing order (so $\beta_{0}>\alpha(*)$ ) (possibly $\left.\alpha_{l(1)}^{j(1)}=\alpha_{l(2)}^{j(2)} \&(j(1), l(1)) \neq(j(2), l(2))\right)$. Now we choose by induction on $m \leq m^{*}$ a condition $q_{m} \in P_{\beta_{m}}$ above $q$, increasing with $m$, where we stipulate $\beta_{m^{*}}=\delta$.

During this definition we "throw a dice" and prove that the probability of success (i.e. $q_{m^{*}} \Vdash " k \in C_{j}$ " for $j<j^{*}$ ) is positive, so there is $q_{m^{*}}$ as desired and hence we get the desired contradiction.

CASE A: $m=0$. Let $q_{0}=q$.

CASE B: $m+1$, and for some $\mathbf{n}<\mathbf{n}^{t}$, we have $\mathbf{n} \in \operatorname{dom}\left(h_{0}^{t}\right)$ and $\zeta$ and: if $j<j^{*}$ and $l<\omega$ then $\alpha_{l}^{j}=\beta_{m} \Rightarrow \mathbf{n}(j)=\mathbf{n} \& p_{l}^{j}=\zeta\left(=h_{0}^{t}(\mathbf{n}(j))(l)\right) \in$ $\left.Q_{\beta_{m}}\right)$. In this case $\operatorname{dom}\left(q_{m+1}\right)=\operatorname{dom}\left(q_{m}\right) \cup\left\{\beta_{m}\right\}$, and

$$
q_{m+1}(\beta)=\left\{\begin{array}{ll}
q_{m}(\beta) & \text { if } \beta<\beta_{m} \\
\zeta & \text { if } \beta=\beta_{m} .
\end{array} \text { (so } \beta \in \operatorname{dom}\left(q_{m}\right)\right),
$$

CASE C: $m+1$ and for some $\mathbf{n}<\mathbf{n}^{t}$, we have $\mathbf{n} \in \operatorname{dom}\left(h_{1}^{t}\right)$ and: $\alpha_{l}^{j}=$ $\beta_{m} \Rightarrow \mathbf{n}(j)=\mathbf{n}$. Work first in $\mathbf{V}\left[G_{P_{\beta_{m}}}\right], q_{m} \in G_{P_{\beta_{m}}}, G_{P_{\beta_{m}}}$ generic over $\mathbf{V}$. The sets

$$
\left\{\lim \left(\underline{\sim}_{l}^{j}\left[G_{P_{\beta_{m}}}\right]\right) \cap\left({ }^{\omega} 2\right)^{\left[h_{2}^{t}(\mathbf{n})\right]}: \alpha_{l}^{j}=\beta_{m}\left(\text { and } l \in\left[n_{k}^{t}, n_{k+1}^{t}\right), j<j^{*}\right)\right\}
$$

are subsets of $\left({ }^{\omega} 2\right)^{\left[h_{2}^{t}(\mathbf{n})\right]}=\left\{\eta \in{ }^{\omega} 2: h_{2}^{t}(\mathbf{n}) \triangleleft \eta\right\}$, and we can define an equivalence relation $E_{m}$ on $\left({ }^{\omega} 2\right)^{\left[h_{2}^{t}(\mathbf{n})\right]}$ :

$$
\begin{array}{cc}
\nu_{1} E_{m} \nu_{2} \quad \text { iff } \quad \nu_{1} \in \lim \left(\underline{\sim}_{l}^{j}\left[G_{P_{\beta_{m}}}\right]\right) \equiv \nu_{2} \in \lim \left(\underline{p}_{l}^{j}\left[G_{P_{\beta_{m}}}\right]\right) \\
\\
\text { whenever } \alpha_{l}^{j}=\beta_{m} .
\end{array}
$$

Clearly $E_{m}$ has finitely many equivalence classes, call them $\left\langle Z_{i}^{m}: i<i_{m}^{*}\right\rangle$; all are Borel (sets of reals), hence measurable; without loss of generality, $\operatorname{Leb}\left(Z_{i}^{m}\right)=0 \Leftrightarrow i \in\left[i_{m}^{\otimes}, i_{m}^{*}\right)$, so clearly $i_{m}^{\otimes}>0$. For each $i<i_{m}^{\otimes}$ there is $r=r_{m, i} \in \underline{Q}_{\beta_{m}}\left[G_{P_{\beta_{m}}}\right]$ such that

$$
\begin{gathered}
\lim \left(p_{l}^{j}\left[G_{P_{\beta_{m}}}\right]\right) \supseteq Z_{i}^{m} \Rightarrow r \geq p_{l}^{j}\left[G_{P_{\beta_{m}}}\right], \\
\lim \left(p_{l}^{i}\left[G_{P_{\beta_{m}}}\right]\right) \cap Z_{i}^{m}=\emptyset \Rightarrow \lim (r) \cap \lim \left(p_{l}^{i}\left[G_{P_{\beta_{m}}}\right]\right)=\emptyset .
\end{gathered}
$$

We can also find a rational $a_{m, i} \in(0,1)_{\mathbb{R}}$ such that

$$
a_{m, i}<\operatorname{Leb}\left(Z_{i}^{m}\right) / 2^{-\lg \left(h_{2}^{t}(\mathbf{n})\right)}<a_{m, i}+\varepsilon /\left(2 i_{m}^{*}\right) .
$$


We can find $q_{m}^{\prime} \in G_{P_{\beta_{m}}}, q_{m} \leq q_{m}^{\prime}$, such that $q_{m}^{\prime}$ forces all this information (so for $Z_{i}^{m}, \underline{\sim}_{m, i}$ we shall have $P_{\beta_{m}}$-names, but $a_{m, i}, i_{m}^{\otimes}, i_{m}^{*}$ are actual objects). We can then find rationals $b_{m, i} \in\left(a_{m, i}, a_{m, i}+\varepsilon / 2\right)$ such that $\sum_{i<i_{m}^{\otimes}} b_{m, i}=1$.

Now we throw a dice choosing $i_{m}<i_{m}^{\otimes}$ with the probability of $i_{m}=i$ being $b_{m, i}$ and define $q_{m+1}$ as:

$$
\begin{gathered}
\operatorname{dom}\left(q_{m+1}\right)=\operatorname{dom}\left(q_{m}^{\prime}\right) \cup\left\{\beta_{m}\right\}, \\
q_{m+1}(\beta)=\left\{\begin{array}{ll}
q_{m}^{\prime}(\beta) & \text { if } \beta<\beta_{m} \\
\underline{\sim}_{m, i_{m}} & \text { if } \beta=\beta_{m} .
\end{array}\left(\text { so } \beta \in \operatorname{dom}\left(q_{m}^{\prime}\right)\right),\right.
\end{gathered}
$$

An important point is that this covers all cases (and in Case B the choice of $(j, l)$ is immaterial) as for each $\beta_{m}$ there is a unique $\mathbf{n}<\mathbf{n}^{t}$ and $l$ such that $\eta_{\beta_{m}}\left\lceil w^{t}=\eta_{\mathbf{n}, l}^{t}\right.$ (see Definitions 2.11(b) and 2.9(i)). Basic probability computations (for $n_{k+1}^{t}-n_{k}^{t}$ independent experiments) show that for each $j$ coming from clause (a), by the law of large numbers, as $k$ was chosen such that $n_{k+1}^{t}-n_{k}^{t}$ is large enough compared to $1 / \varepsilon$ and $j^{*}$, the probability of successes is $>1-1 / j^{*}$, successes meaning $q_{m^{*}} \Vdash$ " $k \in{\underset{\sim}{j}}_{j}$ " (remember that if $j$ comes from clause (b) we always succeed).

REMARK 2.15. In the definition of $t \in \mathcal{T}$ (i.e. 2.9) we can add $\eta_{\mathbf{n}, \omega}^{t} \in w^{t} 2$ (i.e. replace $\left\langle\eta_{\mathbf{n}, l}^{t}: l<\omega\right\rangle$ by $\left.\left\langle\eta_{\mathbf{n}, l}^{t}: l \leq \omega\right\rangle\right)$ and demand

(1) if $\zeta \in w^{t}$ then for every $n<\omega$ large enough, $\eta_{\mathbf{n}, n}^{t}(\zeta)=\eta_{\mathbf{n}, \omega}^{t}(\zeta)$, and in Definition 2.11(d) use $\bar{\alpha}=\left\langle\alpha_{l}: l<\omega\right\rangle$ but this does not help here.

Lemma 2.16. (1) Assume:

(a) $\bar{Q} \in \mathcal{K}^{3}, \bar{Q}=\left\langle P_{\alpha}, Q_{\beta}, A_{\beta}, \mu_{\beta}, \underset{\sim}{r_{\beta}}, \eta_{\beta},\left(\underset{\Xi_{\alpha}^{t}}{\Xi_{t \in T}}: \alpha \leq \alpha^{*}, \beta<\alpha^{*}\right\rangle\right.$,

(b) $A \subseteq \alpha^{*}, \kappa \leq|A|<\lambda$,

(c) $\eta \in\left({ }^{\kappa} 2\right)^{\mathbf{V}} \backslash\left\{\eta_{\beta}: \beta<\alpha^{*}\right\}$,

(d) $(\forall \alpha \in A)\left[\left|A_{\alpha}\right|<\kappa \Rightarrow A_{\alpha} \subseteq A\right]$ and $P_{A}^{\prime}<P_{\alpha^{*}}, \underline{Q}=Q^{A, \bar{Q}}$ is the $P_{\alpha^{*}}$-name from $2.2(\mathrm{~F})(\beta)$ and if $t \in \mathcal{T}$ then $\underset{\sim}{\Xi_{\alpha^{*}}^{t}} \mid \mathbf{V}^{P_{A}^{\prime}}$ is a $P_{A}^{\prime}$-name.

Then there is

$$
\bar{Q}^{+}=\left\langle P_{\alpha}, Q_{\beta}, A_{\beta}, \mu_{\beta}, \tau_{\beta}, \eta_{\beta},\left({\underset{\sim}{\Xi}}_{\alpha}^{t}\right)_{t \in T}: \alpha \leq \alpha^{*}+1, \beta<\alpha^{*}+1\right\rangle
$$

from $\mathcal{K}^{3}$, extending $\bar{Q}$, such that $Q_{\alpha^{*}}=Q, A_{\alpha^{*}}=A, \eta_{\alpha^{*}}=\eta$.

(2) If clauses $(\mathrm{a})+(\mathrm{b})+(\mathrm{c})$ of (1) hold then we can find $A^{\prime}$ such that $A \subseteq A^{\prime} \subseteq \alpha^{*},\left|A^{\prime}\right| \leq(|A|+\kappa)^{\aleph_{0}}$ (which is $<\lambda$ by Hypothesis 2.1) and $\bar{Q}$, $A^{\prime}, \eta$ satisfy $(\mathrm{a})+(\mathrm{b})+(\mathrm{c})+(\mathrm{d})$.

Pro of. Part (2) is easy.

(1) As before the problem is to define $\Xi_{\alpha^{*}+1}^{t}$. We have to satisfy clause (g) of Definition 2.11 for each fixed $t \in \widetilde{\mathcal{T}}$. Let $\mathbf{n}^{*}$ be the unique $\mathbf{n}<\mathbf{n}^{t}$ such that $\eta\left\lceil w^{t}=\eta_{\mathbf{n}, l}^{t}\right.$. If $\mathbf{n}^{*} \in \operatorname{dom}\left(h_{0}^{t}\right)$ or $\left\langle\eta_{\mathbf{n}^{*}, l}^{t}: l<\omega\right\rangle$ is not constant or there is no such $\mathbf{n}^{*}$ then we have nothing to do. So assume that $\alpha_{l}=\alpha^{*}$ and 
$\eta_{\mathbf{n}^{*}, l}^{t}=\eta\left\lceil w^{t}\right.$ for $l<\omega$. Let $\Gamma(\in \mathbf{V})$ be the set of all pairs $\left(\underset{\sim}{r},\left\langle r_{l}: l<\omega\right\rangle\right)$ which satisfy the assumption $(* *)_{x,\left\langle r_{l}: l<\omega\right\rangle}^{\bar{Q}}$ of $2.11(\mathrm{~g})$. In $\mathbf{V}^{P_{\alpha^{*}+1}}$ we have to choose $\underset{\sim}{\Xi_{\alpha^{*}+1}^{t}}$ taking care of all these obligations. We work in $\mathbf{V}^{P_{\alpha^{*}}}$. By assumption (d) and Claim 1.6 it suffices to prove it for $\mathbf{V}^{P_{A}^{\prime}}$ so $Q_{\alpha^{*}}$ is Random $\mathbf{V}^{P_{A}^{\prime}}$ (see 2.3(7)). By 1.7 it is enough to prove condition (B) of 1.7. Suppose it fails. Then there are $\left\langle B_{m}: m<m(*)\right\rangle$, a partition of $\omega$ from $\mathbf{V}^{P_{A}^{\prime}}$, for simplicity $\Xi_{\alpha^{*}}^{t}\left(B_{m}\right)>0$ for $m<m(*)$, and $\left(r^{i},\left\langle\underline{r}_{l}^{i}: l<\omega\right\rangle\right) \in \Gamma$ and $\mathbf{n}(i)=\mathbf{n}^{*}<\mathbf{n}^{t}$ for $i<i^{*}<\omega$ and $\varepsilon^{*}>0$ and $r \in Q_{\alpha^{*}}$ which forces the failure (of (B) of 1.7) for these parameters (the $\varepsilon^{*}$ comes from 1.7). We can assume that $r$ forces $\underline{\sim}^{i} \in G_{Q_{\alpha^{*}}}$ for $i<i^{*}$ (otherwise we can ignore such $\underline{r}^{i}$ as nothing is demanded on them in 2.11(g)). So $r \geq r^{i}$ for $i<i^{*}$ (see $2.2(\mathrm{~F})(\beta))$.

By assumption, for each $i<i^{*}$ we have: for each $r^{\prime} \geq r$ (hence $r^{\prime} \geq r^{i}$ and $r^{\prime} \in$ Random) and $i<i^{*}$ we have

$$
\operatorname{Av}_{\Xi_{\alpha^{*}}^{t}}\left(\left\langle a_{k}^{i}\left(r^{\prime}\right): k<\omega\right\rangle\right) \geq 1-h_{1}^{t}\left(\mathbf{n}^{*}\right)
$$

where (see $2.11(\mathrm{~g})(\otimes))$ we let

$$
a_{k}^{i}\left(r^{\prime}\right)=a_{k}\left(\underset{r}{r}\left\langle\underline{r}_{l}: l<\omega\right\rangle, \bar{n}^{t}\right)=\frac{1}{n_{k+1}^{t}-n_{k}^{t}} \sum_{l \in\left[n_{k}^{t}, n_{k+1}^{t}\right)} \frac{\operatorname{Leb}\left(\lim \left(r^{\prime}\right) \cap \lim \left(r_{l}^{i}\right)\right)}{\operatorname{Leb}\left(\lim \left(r^{\prime}\right)\right)} .
$$

By 1.7 it suffices to prove

Lemma 2.17. Assume $\Xi$ is a finitely additive measure, $\left\langle B_{0}, \ldots, B_{m^{*}-1}\right\rangle$ a partition of $\omega, \Xi\left(B_{m}\right)=a_{m}, i^{*}<\omega$ and $r, r_{l}^{i} \in$ Random for $i<i^{*}, l<\omega$ and $\bar{n}^{*}=\left\langle n_{i}^{*}: i<\omega\right\rangle, n_{i}^{*}<n_{i+1}^{*}<\omega$, are such that

(*) for every $r^{\prime} \in$ Random, $r^{\prime} \geq r$ and $i<i^{*}$ we have $\operatorname{Av} \Xi\left(\left\langle a_{k}^{i}\left(r^{\prime}\right): k<\right.\right.$ $\omega\rangle) \geq b_{i}$ where

$$
\begin{aligned}
a_{k}^{i}\left(r^{\prime}\right) & =a_{k}^{i}\left(r^{\prime},\left\langle r_{l}^{i}: l<\omega\right\rangle, \bar{n}^{*}\right) \\
& =\frac{1}{n_{k+1}^{*}-n_{k}^{*}} \sum_{l=n_{k}^{*}}^{n_{k+1}^{*}-1} \frac{\operatorname{Leb}\left(\lim \left(r^{\prime}\right) \cap \lim \left(r_{l}^{i}\right)\right)}{\operatorname{Leb}\left(\lim \left(r^{\prime}\right)\right)} .
\end{aligned}
$$

Then for each $\varepsilon>0$ and $k^{*}<\omega$ there are a finite $u \subseteq \omega \backslash k^{*}$ and $r^{\prime} \geq r$ such that:

(1) $a_{m}-\varepsilon<\left|u \cap B_{m}\right| /|u|<a_{m}+\varepsilon$ for $m<m^{*}$,

(2) for each $i<i^{*}$ we have

$$
\frac{1}{|u|} \sum_{k \in u} \frac{\mid\left\{l: n_{k}^{*} \leq l<n_{k+1}^{*} \text { and } r^{\prime} \geq r_{l}^{i}\right\} \mid}{n_{k+1}^{*}-n_{k}^{*}} \geq b_{i}-\varepsilon .
$$


Proof. For $i<i^{*}, m<m^{*}$ and $r^{\prime} \geq r$ (from Random) let

$$
c_{i, m}\left(r^{\prime}\right)=\operatorname{Av}_{\Xi\left\lceil B_{m}\right.}\left(\left\langle a_{k}^{i}\left(r^{\prime}\right): k \in B_{m}\right\rangle\right) \in[0,1]_{\mathbb{R}} .
$$

So clearly

$(*)_{1} \quad$ for $r^{\prime} \geq r$ (in Random)

$$
\begin{aligned}
& b_{i} \leq \operatorname{Av}_{\Xi}\left(\left\langle a_{k}^{i}\left(r^{\prime}\right): k<\omega\right\rangle\right)
\end{aligned}
$$

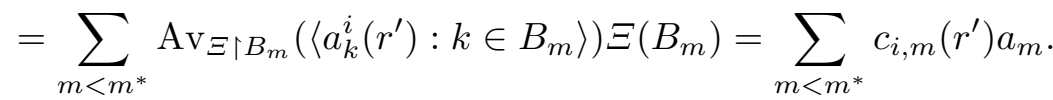

There are $r^{*} \geq r$ and a sequence $\bar{c}=\left\langle c_{i, m}: i<i^{*}, m<m^{*}\right\rangle$ such that:

$(*)_{2} \quad$ (a) $c_{i, m} \in[0,1]_{\mathbb{R}}$

(b) $\sum_{m<m^{*}} c_{m, i} a_{m} \geq b_{i}$

(c) for every $r^{\prime} \geq r^{*}$ there is $r^{\prime \prime} \geq r^{\prime}$ such that

$$
\left(\forall i<i^{*}\right)\left(\forall m<m^{*}\right)\left[c_{i, m}-\varepsilon<c_{i, m}\left(r^{\prime \prime}\right)<c_{i, m}+\varepsilon\right] .
$$

[Why? Let $k^{*}<\omega$ be such that $1 / k^{*}<\varepsilon /\left(10 i^{*} m^{*}\right)$ (so $k^{*}>0$ ). Let $\Gamma=\left\{\bar{c}: \bar{c}=\left\langle c_{i, m}: i<i^{*}, m<m^{*}\right\rangle, c_{i, m} \in[0,1]_{\mathbb{R}}\right.$ and $k^{*} c_{i, m}$ is an integer and $\left.\sum_{m<m^{*}} c_{i, m} a_{m} \geq b_{i}\right\}$ for $i<i^{*}$. Clearly $\Gamma$ is finite and let us list it as $\left\langle\bar{c}^{s}: s<s^{*}\right\rangle$. We try to choose by induction on $s \leq s^{*}$ a condition $r_{s} \in$ Random such that $r_{0}=r, r_{s} \leq r_{s+1}$, and if $\bar{c}^{s}$ satisfies $(*)_{2}(\mathrm{a})+(\mathrm{b})$ then for no $r^{\prime \prime} \geq r_{s+1}$ do we have

$$
\left(\forall i<i^{*}\right)\left(\forall m<m^{*}\right)\left[c_{i, m}^{s}-\varepsilon<c_{i, m}\left(r^{\prime \prime}\right)<c_{i, m}^{s}+\varepsilon\right] .
$$

For $s=0$ we have no problem. If we succeed in arriving at $r_{s^{*}}$, for $i<i^{*}$, $m<m^{*}$ we can define $c_{i, m}^{*} \in\left\{l / k^{*}: l \in\left\{0, \ldots, k^{*}\right\}\right\}$ such that $c_{i, m}\left(r_{s^{*}}\right) \leq$ $c_{i, m}^{*}<c_{i, m}\left(r_{s^{*}}\right)+\varepsilon /\left(10 i^{*} m^{*}\right)$. By $(*)_{1}$ we have $b_{i} \leq \sum_{m<m^{*}} c_{i, m}\left(r_{s^{*}}^{*}\right) a_{m}$. Clearly

$$
\sum_{m<m^{*}} c_{i, m}^{s} a_{m} \geq \sum_{m<m^{*}} c_{i, m}\left(r_{s^{*}}^{*}\right) a_{m}
$$

so $\bar{c}^{*}=\left\langle c_{i, m}^{*}: i<i^{*}, m<m^{*}\right\rangle$ satisfies $(*)_{2}(\mathrm{a})+(\mathrm{b})$ and $\bar{c}^{*} \in \Gamma$, hence $\bar{c}^{*}=\bar{c}^{s}$ for some $s<s^{*}$. But then $r^{*}$ contradicts the choice of $r_{s+1}$. Also by the above $\Gamma \neq \emptyset$. So we are necessarily stuck at some $s<s^{*}$, i.e. cannot find $r_{s+1}$ as required. As $\bar{c}^{+}$satisfies $(*)_{2}(\mathrm{a})+(\mathrm{b})$, this means that $r_{s}, \bar{c}^{s}$ as needed in $(*)_{2}$, so $r^{*}, \bar{c}$ as required exist.]

Let $k^{*}<\omega$ be given. Now choose $s^{*}<\omega$ large enough and try to choose, by induction on $s \leq s^{*}$, a condition $r_{s} \in$ Random and natural numbers $\left(m_{s}, k_{s}\right)$ (flipping coins along the way) such that:

$(*)_{3} \quad$ (a) $r_{0}=r^{*}$,

(b) $r_{s+1} \geq r_{s}$,

(c) $c_{i, m}-\varepsilon<c_{i, m}\left(r_{s}\right)<c_{i, m}+\varepsilon$ for $i<i^{*}, m<m^{*}$,

(d) $k_{s}>k^{*}, k_{s+1}>k_{s}$,

(e) $k_{s} \in B_{m_{s}}$. 
In stage $s$, given $r_{s}$, we define $r_{s+1}, i_{s}, m_{s}, k_{s}$ as follows: choose $m_{s}<m^{*}$ randomly with the probability of $m_{s}=m$ being $a_{m}$. Next we can find a finite set $u_{s} \subseteq B_{m_{s}}$ with $\min \left(u_{s}\right)>\max \left\{k^{*}+1, k_{s_{1}}+1: s_{1}<s\right\}$ such that

$(*) \quad$ if $\quad i<i^{*} \quad$ then $\quad c_{i, m_{s}}-\varepsilon / 2<\frac{1}{\left|u_{s}\right|} \sum_{k \in u_{s}} a_{k}^{i}\left(r_{s}\right)<c_{i, m_{s}}+\varepsilon / 2$.

We define an equivalence relation $\mathbf{e}_{s}$ on $\lim \left(r_{s}\right)$ :

$\eta_{1} \mathbf{e}_{s} \eta_{2} \quad$ iff $\quad\left(\forall i<i^{*}\right)\left(\forall k \in u_{s}\right)\left(\forall l \in\left[n_{k}^{*}, n_{k+1}^{*}\right)\right)\left[\eta_{1} \in \lim \left(r_{l}^{i}\right) \equiv \eta_{2} \in \lim \left(r_{l}^{i}\right)\right]$.

The number of $\mathbf{e}_{s}$-equivalence classes is finite, and if $Y \in \lim \left(r_{s}\right) / \mathbf{e}_{s}$ satisfies $\operatorname{Leb}(Y)>0$ choose $r_{s, Y} \in$ Random such that $\lim \left(r_{s, Y}\right) \subseteq Y$ and $r_{s, Y}$ satisfies clause $(\mathrm{c})$ of $(*)_{3}$ (possible by clause $(\mathrm{c})$ of $\left.(*)_{2}\right)$. Now choose $r_{s+1}$ among $\left\{r_{s, Y}: Y \in \lim \left(r_{s}\right) / \mathbf{e}_{s}\right.$ and $\left.\operatorname{Leb}(Y)>0\right\}$, with the probability of $r_{s, Y}$ being $\operatorname{Leb}(Y) / \operatorname{Leb}\left(\lim \left(r_{s}\right)\right)$. Lastly choose $k_{s} \in u_{s}$, with all $k \in u_{s}$ having the same probability.

Now the expected value, assuming $m_{s}=m$, of

$$
\frac{1}{n_{k_{s}+1}^{*}-n_{k_{s}}^{*}} \mid\left\{l: n_{k_{s}}^{*} \leq l<n_{k_{s}+1}^{*} \text { and } r_{s+1} \geq r_{l}^{i}\right\} \mid
$$

belongs to the interval $\left(c_{i, m}-\varepsilon / 2, c_{i, m}+\varepsilon / 2\right)$, because the expected value of

$$
\frac{1}{\left|u_{s}\right|} \sum_{k \in u_{s}} \frac{1}{n_{k+1}^{*}-n_{k}^{*}} \mid\left\{l: n_{k}^{*} \leq l<n_{k+1}^{*} \text { and } r_{s+1} \geq r_{l}^{i}\right\} \mid
$$

is in this interval (as

$$
\sum\left\{\operatorname{Leb}(Y): Y \in \lim \left(r_{s}\right) / \mathbf{e}_{s}, r_{s, Y} \geq r_{l}^{i}\right\}=\frac{\operatorname{Leb}\left(\lim \left(r_{s}\right) \cap \lim \left(r_{l}^{i}\right)\right)}{\operatorname{Leb}\left(\lim \left(r_{s}\right)\right)}
$$

and see the choice of $\left.a_{k}^{i}(-)\right)$.

Let $r^{\prime}=r_{s^{*}}, u=\left\{k_{s}: s \leq s^{*}\right\}$. Hence the expected value of

$$
\frac{1}{|u|} \sum_{k \in u} \frac{1}{n_{k+1}^{*}-n_{k}^{*}} \mid\left\{l: n_{k}^{*} \leq l<n_{k+1}^{*} \text { and } r^{\prime} \geq r_{l}^{i}\right\} \mid
$$

is $\geq \sum a_{m}\left(c_{i, m}-\varepsilon / 2\right) \geq b_{i}-\varepsilon / 2$.

As $s^{*}$ is large enough with high probability (though just positive probability suffices), $\left(r_{s^{*}},\left\{k_{s}: s<s^{*}\right\}\right)$ are as required for $\left(r^{\prime}, u\right)$; note: we do not know the variance but we have a bound for it not depending on $s$.

(2) Straightforward.

This completes the proof of Lemma 2.16.

The following is needed later to show that there are enough cases of the definition of $t$ with clause (g) of Definition 2.11 being non-trivial (i.e. $(* *)$ there holds). 
Lemma 2.18. Assume:

(a) $\Xi$ is a finitely additive measure on $\omega$ and $b \in(0,1]_{\mathbb{R}}$,

(b) $n_{k}^{*}<\omega($ for $k<\omega), n_{k}^{*}<n_{k+1}^{*}$, and $\lim \left(n_{k+1}^{*}-n_{k}^{*}\right)=\infty$,

(c) $r^{*}, r_{l} \in$ Random are such that

(*) $(\forall l<\omega)\left[\operatorname{Leb}\left(\lim \left(r^{*}\right) \cap \lim \left(r_{l}\right)\right) / \operatorname{Leb}\left(\lim \left(r^{*}\right)\right) \geq b\right]$.

Then for some $r^{\otimes} \geq r^{*}$ we have:

$\otimes_{r} \otimes \quad$ for every $r^{\prime} \geq r^{\otimes}$ we have $\operatorname{Av}_{\Xi}\left(\left\langle a\left(r^{\prime}, k\right): k<\omega\right\rangle\right) \geq b$ where $a_{k}\left(r^{\prime}\right)=$ $a\left(r^{\prime}, k\right)=a_{k}\left(\lim r^{\prime}\right)$ and for any Borel set $X \subseteq{ }^{\omega} 2$ we let

$$
a_{k}(X)=\frac{1}{n_{k+1}^{*}-n_{k}^{*}} \sum_{l \in\left[n_{k}^{*}, n_{k+1}^{*}\right)} \frac{\operatorname{Leb}\left(X \cap \lim \left(r_{l}\right)\right)}{\operatorname{Leb}(X)} .
$$

Proof. Let

$$
\mathcal{I}=\left\{r \in \text { Random }: r \geq r^{*} \text { and } \operatorname{Av}_{\Xi}\left(\left\langle a_{k}\left(r^{\prime}\right): k<\omega\right\rangle\right)<b\right\} .
$$

If $\mathcal{I}$ is not dense above $r^{*}$ there is $r^{\otimes} \geq r^{*}$ (in Random) such that for every $r \geq r^{\otimes}, r \notin \mathcal{I}$ so $r^{\otimes}$ is as required; so assume toward contradiction that $\mathcal{I}$ is dense above $r^{*}$. There is a maximal antichain $\mathcal{I}_{1}=\left\{s_{i}: i<i^{*}\right\}$ $\subseteq \mathcal{I}$ (maximal among those $\subseteq \mathcal{I}$ ); it is a maximal antichain above $r^{*}$ as $r \in \mathcal{I} \Rightarrow r \geq r^{*}$ and by the previous sentence. Hence $\operatorname{Leb}\left(\lim \left(r^{*}\right)\right)=$ $\sum_{i<i^{*}} \operatorname{Leb}\left(\lim \left(s_{i}\right)\right)$; of course $\left|i^{*}\right| \leq \aleph_{0}$ as Random satisfies c.c.c. so we can assume $i^{*} \leq \omega$.

For any $j<i^{*}$ let $s^{j}=\bigcup_{i<j} s_{i}$. Note then $\lim \left(\bigcup_{m<i} s_{m}\right)=\bigcup_{m<i} \lim \left(s_{m}\right)$ and

hence

$$
a_{k}\left(s^{j}\right)=a_{k}\left(\bigcup_{m<i} s_{m}\right)=\sum_{i<j} \frac{\operatorname{Leb}\left(s_{i}\right)}{\operatorname{Leb}\left(\bigcup_{m<j} s_{m}\right)} a_{k}\left(s_{i}\right),
$$

$$
\begin{aligned}
\operatorname{Av}_{\Xi}\left(\left\langlea_{k}\left(s^{j}\right)\right.\right. & : k<\omega\rangle) \\
& =\operatorname{Av} \Xi_{\Xi}\left(\left\langle a_{k}\left(\bigcup_{m<j} s_{m}\right): k<\omega\right\rangle\right) \\
& =\sum_{i<j} \frac{\operatorname{Leb}\left(s_{i}\right)}{\operatorname{Leb}\left(\bigcup_{m<j} s_{m}\right)} \operatorname{Av} \Xi\left(\left\langle a_{k}\left(s_{i}\right): k<\omega\right\rangle\right) \\
& \leq \frac{\operatorname{Leb}\left(\lim \left(s_{0}\right)\right)}{\operatorname{Leb}\left(\lim \left(\bigcup_{i<j} s_{i}\right)\right)}(b-\varepsilon)+\sum_{0<i<j} \frac{\operatorname{Leb}\left(\lim \left(s_{i}\right)\right)}{\operatorname{Leb}\left(\lim \left(\bigcup_{m<j} s_{m}\right)\right)} b \\
& =b-\operatorname{Leb}\left(\lim \left(s_{0}\right)\right) \varepsilon
\end{aligned}
$$

where $\varepsilon=b-\operatorname{Av}_{\Xi}\left(\left\langle a_{k}\left(s_{0}\right): k<\omega\right\rangle\right)$ so $\varepsilon>0$.

Let $j$ be large enough such that

$$
\frac{\operatorname{Leb}\left(\lim \left(r^{*}\right) \backslash \lim \left(s^{j}\right)\right)}{\operatorname{Leb}\left(\lim \left(r^{*}\right)\right)}<\operatorname{Leb}\left(\lim \left(s_{0}\right)\right) \varepsilon .
$$


So

$$
\begin{aligned}
\operatorname{Av}_{\Xi}\left(\left\langle a_{k}\left(r^{*}\right): k<\omega\right\rangle\right) & \\
= & \frac{\operatorname{Leb}\left(\lim \left(r^{*}\right) \backslash \lim \left(s^{j}\right)\right)}{\operatorname{Leb}\left(\lim \left(r^{*}\right)\right)} \operatorname{Av} \Xi\left(\left\langle a_{k}\left(\lim \left(r^{*}\right) \backslash \lim \left(s^{j}\right)\right): k<\omega\right\rangle\right) \\
& +\frac{\operatorname{Leb}\left(\lim \left(s^{j}\right)\right)}{\operatorname{Leb}\left(\lim \left(r^{*}\right)\right)} \operatorname{Av} \Xi\left(\left\langle a_{k}\left(s^{j}\right): k<\omega\right\rangle\right) \\
\leq & \frac{\operatorname{Leb}\left(\lim \left(r^{*}\right) \backslash \lim \left(s^{j}\right)\right)}{\operatorname{Leb}\left(r^{*}\right)} 1+\frac{\operatorname{Leb}\left(\lim \left(s^{j}\right)\right)}{\operatorname{Leb}\left(\lim \left(r^{*}\right)\right)}\left(b-\operatorname{Leb}\left(\lim \left(s_{0}\right)\right) \varepsilon\right) \\
< & \operatorname{Leb}\left(\lim \left(s_{0}\right)\right) \varepsilon+\left(b-\operatorname{Leb}\left(\lim \left(s_{0}\right)\right) \varepsilon\right)=b,
\end{aligned}
$$

contradicting assumption (c).

Claim 2.19. Assume:

(a) $\bar{Q} \in \mathcal{K}^{3}, \bar{Q}=\left\langle P_{\alpha}, Q_{\beta}, A_{\beta}, \mu_{\beta}, \underset{\sim}{r_{\beta}}, \eta_{\beta},\left(\Xi_{\alpha}^{t}\right)_{t \in T}: \alpha \leq \alpha^{*}, \beta<\alpha^{*}\right\rangle$,

(b) $A \subseteq \alpha^{*}$ and $\left({ }^{6}\right)|A|<\kappa$ and $\mu<\kappa$ are such that $\beta \in A \&\left|A_{\beta}\right|<\kappa$ $\Rightarrow A_{\beta} \subseteq A$,

(c) $\eta \in{ }^{\kappa} 2 \backslash\left\{\eta_{\beta}: \beta<\alpha^{*}\right\}$,

(d) $Q$ is a $P_{\alpha^{*}}$-name of a forcing notion with set of elements $\mu$, and is really definable in $\mathbf{V}\left[\left\langle\tau_{\alpha}: \alpha \in A\right\rangle\right]$ from $\left\langle\tau_{\alpha}: \alpha \in A\right\rangle$ and parameters from $\mathbf{V}$ (we can even assume the truth value of $\zeta_{1}<_{\theta} \zeta_{2}$ for $\zeta_{1}<\zeta_{2}$ is as in 2.3(5)).

Then there is

$$
\bar{Q}^{+}=\left\langle P \alpha, Q_{\alpha}, A_{\beta}, \mu_{\beta}, \tau_{\beta}, \eta_{\beta},\left(\Xi_{\sim}^{t}\right)_{t \in \mathcal{T}}: \alpha \leq \alpha^{*}+1, \beta<\alpha^{*}+1\right\rangle
$$

from $\mathcal{K}^{3}$ extending $\bar{Q}$ such that $\underline{Q}_{\alpha^{*}}=Q, A_{\alpha^{*}}=A, \eta_{\alpha^{*}}=\eta$ and $\mu_{\alpha^{*}}=\mu$.

\section{Proof. Straight.}

Remark 2.20. If $Q$ is the Cohen forcing we can make one step toward $\left\{A \subseteq \omega: \Xi_{\alpha^{*}+1}^{t}(A)=1\right\}$ being a selective filter, even simultaneously for all $\omega$-sequences of members, but not needed at present.

3. Continuation of the proof of Theorem 0.1. We need the following lemma.

Lemma 3.1. Suppose that $\bar{\varepsilon}=\left\langle\varepsilon_{l}: l<\omega\right\rangle$ is a sequence of positive reals and $\bar{Q} \in \mathcal{K}^{3}$ has length $\alpha$. The following set $\mathcal{I}_{\bar{\varepsilon}} \subseteq P_{\alpha}$ is dense:

$\mathcal{I}_{\bar{\varepsilon}}=\left\{p \in P_{\alpha}^{\prime}:\right.$ there are $m$ and $\alpha_{l}, \nu_{l}($ for $l<m)$ such that
(a) $\operatorname{dom}(p)=\left\{\alpha_{0}, \ldots, \alpha_{m-1}\right\}, \alpha_{0}>\alpha_{1}>\ldots>\alpha_{m-1}$,
(b) if $\left|A_{\alpha_{l}}\right|<\kappa$, then $p\left(\alpha_{l}\right)$ is an ordinal,

$\left({ }^{6}\right)$ If " $(\forall \alpha<\kappa)\left(\left.|\alpha|\right|^{\aleph_{0}}<\kappa\right)$ " is added to 2.1 , it is natural to restrict ourselves to the case $P_{A}^{\prime}<0 P_{\alpha^{*}}$ as the parallel of 2.16(2) holds. 
(c) if $Q_{\alpha_{l}}$ is a partial random, i.e. $\left|A_{\alpha_{l}}\right| \geq \kappa$, then $\Vdash_{P_{\alpha_{l}}}$ " $p\left(\alpha_{l}\right) \subseteq\left({ }^{\omega} 2\right)^{\left[\nu_{l}\right]}$ and $\left.\left.\operatorname{Leb}\left(\lim \left(p\left(\alpha_{l}\right)\right)\right) \geq\left(1-\varepsilon_{l}\right) / 2^{\lg \left(\nu_{l}\right)}\right)\right\}$.

Proof. By induction on $\alpha$ for all possible $\bar{\varepsilon}$.

Discussion 3.2. (1) By the previous sections it follows that it is enough to prove that if $\bar{Q} \in \mathcal{K}^{3}, P_{\alpha}=\operatorname{Lim}(\bar{Q})$, then in $\mathbf{V}^{P_{\alpha}}$ the following sufficient condition holds:

$(* *)_{\bar{Q}} \quad$ In $\mathbf{V}^{P_{\alpha}}$, there is no perfect tree $T \subseteq{ }^{\omega>} 2, m \in \omega$ and $E \in[\lambda]^{\kappa^{+}}$ such that $T \subseteq \operatorname{tree}_{m}\left[\bar{a}^{\alpha}\right]$ for all $\alpha \in E$.

(2) Note that if we just want to prove $\Vdash_{P_{\alpha}}$ "b $\leq \kappa$ " life is easier: $\Xi_{\alpha}^{t}$ can be chosen a zero-one measure (so essentially an ultrafilter) and for $\alpha<\lambda$ we interpret the forcing notion $Q_{\alpha}$ as $(\omega>\omega, \triangleleft)$ with generic real $\eta_{\alpha}$ and replace below $(* *)_{\bar{Q}}$ by

$(* *)_{\bar{Q}}^{+} \quad$ in $\mathbf{V}^{P_{\alpha}}$, there is no $\eta^{*} \in{ }^{\omega} \omega$ such that $\left\{\alpha<\lambda:(\forall l<\omega)\left(\eta_{\alpha}(l) \leq\right.\right.$ $\left.\left.\eta^{*}(l)\right)\right\}$ has cardinality $\geq \kappa^{+}$.

In the proof below, $\underset{\sim}{T}$ is replaced by $\underset{\eta}{ }$, and $p_{\zeta}^{\prime}\left(\alpha_{\zeta}\right)$ is $s^{*}\langle\zeta\rangle$.

(3) We can make the requirements on the $\Delta$-system stronger: make it indiscernible also over some $A \subseteq \alpha$ of cardinality $<\kappa$, where $\underset{\sim}{T}$ is a $P_{A}^{\prime}$-name, $p^{*} \in P_{A}^{\prime}$, and assume the heart is $\subseteq A$. small.

(4) Here the existence of $h_{2}^{t}$ helps; we can use 3.1 with $\sum_{l<\omega} \varepsilon_{l}$ very

Lemma 3.3. If $P_{\alpha}=\operatorname{Lim}(\bar{Q}), \alpha=\lg (\bar{Q})$ and $\bar{Q} \in \mathcal{K}^{3}$, then $(* *)_{\bar{Q}}$ from 2.7 holds.

P r o of. Suppose $p^{*} \vdash_{P_{\alpha}} \underset{\sim}{T}, m \underset{\sim}{E}$ form a counterexample to $(* *)_{\bar{Q}}$ "; we can assume $p^{*} \in P_{\alpha}^{\prime}$. Let $\bar{\varepsilon}=\left\langle\varepsilon_{l}: l<\omega\right\rangle$ be such that $\varepsilon_{l} \in(0,1)_{\mathbb{R}}$ and $\sum_{l<\omega} \sqrt{\varepsilon_{l}}<1 / 10$. For each $\zeta<\kappa^{+}$let $p_{\zeta} \geq p^{*}$ be such that $p_{\zeta} \in \mathcal{I}_{\bar{\varepsilon}}\left(\subseteq P_{\alpha}^{\prime}\right)$ witnessed by $\left\langle\nu_{\beta}^{\zeta}: \beta \in \operatorname{dom}\left(p_{\zeta}\right)\right.$ and $\left.\left|A_{\beta}\right| \geq \kappa\right\rangle$ (on $\mathcal{I}_{\bar{\varepsilon}}$ see 3.1) and

$$
p_{\zeta} \Vdash_{P_{\alpha}} \text { " } \alpha_{\zeta} \text { is the } \zeta \text { th element of } \underset{\sim}{E} \text { ". }
$$

So clearly $\alpha_{\zeta}<\lambda$. By thinning out, we can assume that:

- $\operatorname{dom}\left(p_{\zeta}\right)=\left\{\gamma_{i}^{\zeta}: i<i^{*}\right\}$ with $\gamma_{i}^{\zeta}$ increasing with $i$; let $v_{0}^{\zeta}=\left\{i<i^{*}\right.$ : $\left.\left|A_{\gamma_{i}^{\zeta}}\right|<\kappa\right\}$; then $v_{0}^{\zeta}=v_{0}$ is fixed for all $\zeta<\kappa^{+}$, and let $v_{1}=i^{*} \backslash v_{0}$,

- $\operatorname{dom}\left(p_{\zeta}\right)\left(\zeta<\kappa^{+}\right)$form a $\Delta$-system with heart $\Delta$, so $\Delta \supseteq \operatorname{dom}\left(p^{*}\right)$,

- $\alpha_{\zeta} \in \operatorname{dom}\left(p_{\zeta}\right), \alpha_{\zeta}=\gamma_{z}^{\zeta}$ for a fixed $z<i^{*}$,

- $\left(\operatorname{dom}\left(p_{\zeta}\right), \Delta,<\right)$ are pairwise isomorphic for $\zeta<\kappa^{+}$,

- if $i \in v_{0}$, then $p_{\zeta}\left(\gamma_{i}^{\zeta}\right)=\gamma_{i}$ for $\zeta<\kappa^{+}$, of $\left.\mathcal{I}_{\bar{\varepsilon}}\right)$,

- if $i \in v_{1}$, then $\nu_{\gamma_{i}^{\zeta}}^{\zeta}=\nu_{i}$ (recall that $\nu_{\gamma_{i}^{\zeta}}^{\zeta} \in \omega>2$ is given by the definition 
- $p_{\zeta}\left(\alpha_{\zeta}\right)=s^{*}$ for $\zeta<\kappa^{+}$with $s^{*}=\left\langle\left(n_{l}, a_{l}\right): l<m^{*}\right\rangle$; we can assume $m^{*}>m$ (where $m$ is from the "counterexample to $(* *)_{\bar{Q}}$ ") and $m^{*}>10$,

- for each $i<i^{*}$ the sequence $\left\langle\gamma_{i}^{\zeta}: \zeta<\kappa^{+}\right\rangle$is constant or strictly increasing,

- the sequence $\left\langle\alpha_{\zeta}: \zeta<\kappa^{+}\right\rangle$is with no repetitions (since if $p_{\zeta_{1}}, p_{\zeta_{2}}$ are compatible and $\zeta_{1}<\zeta_{2}<\lambda$ then $\left.\alpha_{\zeta_{1}} \neq \alpha_{\zeta_{2}}\right)$,

- if $\gamma_{i(1)}^{\zeta(1)}=\gamma_{i(2)}^{\zeta(2)}$ then $i(1)=i(2)\left(\right.$ when $\zeta(1), \zeta(2)<\kappa_{3}^{+}$and $\left.i(1), i(2)<i^{*}\right)$.

Now we are interested only in the first $\omega$ conditions, i.e. we consider $\zeta<$ $\omega$. For every such $\zeta$ let $p_{\zeta}^{\prime} \geq p_{\zeta}$ be such that $\operatorname{dom}\left(p_{\zeta}^{\prime}\right)=\operatorname{dom}\left(p_{\zeta}\right), p_{\zeta}^{\prime}(\gamma)=$ $p_{\zeta}(\gamma)$ except for $\gamma=\alpha_{\zeta}$ in which case we extend $p_{\zeta}\left(\alpha_{\zeta}\right)=s^{*}$ in the following way. We put $\lg \left(p_{\zeta}^{\prime}\left(\alpha_{\zeta}\right)\right)=\lg \left(s^{*}\right)+1=m^{*}+1, p_{\zeta}^{\prime}\left(\alpha_{\zeta}\right)=s^{*}\left\langle\left(j_{\zeta}^{0}, a_{\zeta}\right)\right\rangle$. Before we define $j_{\zeta}^{0}, a_{\zeta}$ choose an increasing sequence of integers $\bar{s}=\left\langle s_{l}: l<\omega\right\rangle$, $s_{0}=0$, such that $\left.s_{k+1}-s_{k}=\mid{ }^{j_{k}} 2\right]^{2^{j_{k}}\left(1-8^{-m^{*}}\right)} \mid$ (i.e. it is the number of subsets of ${ }^{j_{k}} 2$ with $2^{j_{k}}\left(1-8^{-m^{*}}\right)$ elements), where $j^{*}=3 n_{m^{*}-1}+1$ (i.e. we define $j^{*}$ from the first coordinate in the last pair in $s^{*}$ ) and we let $j_{k}=j^{*}+k !$ !, and $j_{\zeta}^{0}=j_{k}$ when $\zeta \in\left[s_{k}, s_{k+1}\right)$. Now for $\zeta \in\left[s_{k}, s_{k+1}\right)$ define $a_{\zeta}$ such that

$$
\left\{a_{\zeta}: \zeta \in\left[s_{k}, s_{k+1}\right)\right\}=\left[{ }^{j_{k}} 2\right]^{2^{j_{k}}\left(1-8^{-m^{*}}\right)}
$$

(so necessarily without repetitions). For $\varepsilon^{*}>0$ we define a $P_{\alpha}$-name by

$$
\underset{\sim}{A_{\varepsilon^{*}}}=\left\{k<\omega:\left|\left\{\zeta \in\left[s_{k}, s_{k+1}\right): p_{\zeta}^{\prime} \in{\underset{\sim}{P_{\alpha}}}\right\}\right| /\left(s_{k+1}-s_{k}\right)>\varepsilon^{*}\right\} .
$$

For the proof of 3.3 we need:

Claim 3.4. There is a condition $p^{\otimes} \geq p^{*}$ which forces that for some $\varepsilon^{*}>0$ the set $A_{\varepsilon^{*}}$ is infinite.

Proof. Choose $\varepsilon^{*}>0$ small enough. First we define a suitable blueprint $t \in \mathcal{T}$,

$$
t=\left(w^{t}, \mathbf{n}^{t}, \mathbf{m}^{t}, \bar{\eta}^{t}, h_{0}^{t}, h_{1}^{t}, h_{2}^{t}, \bar{n}^{t}\right)
$$

Let

$$
\begin{aligned}
w^{t}=\left\{\min \left\{\beta<\kappa: \eta_{\gamma_{i(1)}^{\zeta(1)}}(\beta) \neq \eta_{\gamma_{i(2)}^{\zeta(2)}}(\beta)\right\}: \zeta(1), \zeta(2)<\omega\right. \text { and } & \\
& \left.i(1), i(2)<i^{*} \text { and } \gamma_{i(1)}^{\zeta(1)} \neq \gamma_{i(2)}^{\zeta(2)}\right\} .
\end{aligned}
$$

Let $\mathbf{n}^{t}=i^{*}, \operatorname{dom}\left(h_{0}^{t}\right)=v_{0}, \operatorname{dom}\left(h_{1}^{t}\right)=\operatorname{dom}\left(h_{2}^{t}\right)=v_{1}$ and $n_{l}^{t}=s_{l}$. If $\mathbf{n} \in v_{0}$, then $h_{0}^{t}(\mathbf{n})(l)=\gamma_{\mathbf{n}}$ and $\eta_{\mathbf{n}, \zeta}^{t}=\eta_{\gamma_{\mathbf{n}}^{\zeta}}\left\lceil w^{t}\right.$. If $\mathbf{n} \in v_{1}$, then $h_{1}^{t}(\mathbf{n})=\varepsilon_{n}$ and $h_{2}^{t}(\mathbf{n})=\nu_{\mathbf{n}}$.

We now define a condition $p^{\otimes}$; it will be in $P_{\alpha}, \operatorname{dom}\left(p^{\otimes}\right)=\Delta, p^{*} \leq p^{\otimes}$; remember $\operatorname{dom}\left(p^{*}\right) \subseteq \Delta$ as for each $\zeta$ we have $p^{*} \leq p_{\zeta}$. If $\gamma \in \Delta$ then for 
some $\mathbf{n}<\mathbf{n}^{t}$, we have $\bigwedge_{\zeta<\omega} \gamma_{\mathbf{n}}^{\zeta}=\gamma$. If $\mathbf{n} \in v_{0}$ we let $p^{\otimes}(\gamma)=h_{0}^{t}(\mathbf{n})$, so trivially in $\mathbf{V}^{P_{\gamma}}$,

$$
p^{\otimes}(\gamma) \Vdash_{Q_{\gamma}} “ \Xi_{\sim+1}^{t}\left(\left\{\zeta<\omega: h_{0}^{t}(\mathbf{n}) \in G_{Q_{\gamma}}\right\}\right)=1 \text { if } \mathbf{n} \in \operatorname{dom}\left(h_{0}^{t}\right)\left(=v_{0}\right) " .
$$

If $\mathbf{n} \in v_{1}$, then define a $P_{\gamma}$-name for a member of $Q_{\gamma}$ as follows. Consider $\underline{r}_{\zeta}^{\mathbf{n}}=p_{\sim}^{p}(\gamma)$ for $\zeta<\omega$. Let $\underset{\sim}{r}$ be the member $\left({ }^{\omega} 2\right)^{\left[h_{2}^{t}(n)\right]}$ of $Q_{\gamma}$. If we work in $\mathbf{V}^{P_{A_{\alpha}}^{\prime}}$, by Lemma 2.18 there is ${\underset{r}{r}}_{\gamma}^{*} \geq \underset{\sim}{r}$ from $\underline{Q}_{\gamma}$ such that for every $r^{\prime} \geq r_{\gamma}^{*}$ in $Q_{\gamma}$ we have

$$
\begin{aligned}
&(* *)_{r^{\prime}, \varepsilon} \quad \operatorname{Av}_{\Xi_{\alpha}^{t}}\left(\left\langle a_{k}^{\mathbf{n}}\left(r^{\prime}\right): k<\omega\right\rangle\right) \geq 1-h_{1}^{t}(\mathbf{n})=1-\varepsilon_{\mathbf{n}} \text { where } \\
& a_{k}^{\mathbf{n}}\left(r^{\prime}\right):=\frac{1}{n_{k+1}^{t}-n_{k}^{t}} \sum_{l \in\left[n_{k}^{t}, n_{k+1}^{t}\right)} \frac{\operatorname{Leb}\left(\lim \left(r^{\prime}\right) \cap \lim \left(r_{l}^{\mathbf{n}}\right)\right)}{\operatorname{Leb}\left(\lim \left(r^{\prime}\right)\right)} .
\end{aligned}
$$

Hence the assumption of condition (g) in Definition 2.11 holds, and so in $\mathbf{V}^{P_{\gamma}}$ we have

$$
\begin{aligned}
& r_{\gamma}^{*} \Vdash_{Q_{\gamma}} " \operatorname{Av}_{\Xi_{\gamma+1}^{t}}\left(\left\langle\left|\left\{l \in\left[n_{k+1}^{t}-n_{k}^{t}\right): p_{l}(\gamma) \in G_{Q_{\gamma}}\right\}\right| /\left(n_{k+1}^{t}-n_{k}^{t}\right): k \in \omega\right\rangle\right) \\
& \geq 1-\varepsilon_{\mathbf{n}} " .
\end{aligned}
$$

So there is a $P_{\gamma}$-name ${\underset{\sim}{\gamma}}_{\gamma}^{*}$ of such a condition. In this case let $p^{\otimes}(\gamma)={\underset{\sim}{\gamma}}_{\gamma}^{*}$, so we have finished defining $p^{\otimes}$; clearly it has the right domain.

Now suppose that $\mathbf{n}<\mathbf{n}^{t}$ with $\mathbf{n} \in v_{1}$ is such that $\gamma_{\mathbf{n}}^{\zeta} \notin \Delta$. Define $\bar{\beta}=\left\langle\beta_{\zeta}: \zeta<\omega\right\rangle, \beta_{\zeta}=\gamma_{\mathbf{n}}^{\zeta}$. Then $\bar{\beta}$ satisfies $(t, \mathbf{n})$ for $\bar{Q}$. By our assumption the assumption of clause (f) in Definition 2.11 is satisfied, hence in $\mathbf{V}^{P_{\alpha}}$, for any $\varepsilon>0$,

$\Vdash_{P_{\alpha}} " \Xi_{\sim}^{t}\left(\left\{k: \frac{\left|\left\{l \in\left[n_{k}^{t}, n_{k+1}^{t}\right): p_{l}\left(\gamma_{n}^{l}\right) \in G_{Q_{\gamma_{n}^{l}}}\right\}\right|}{n_{k+1}^{t}-n_{k}^{t}} \geq\left(1-\varepsilon_{\mathbf{n}}\right)(1-\varepsilon)\right\}\right)=1 "$.

Now for each $\mathbf{n} \in v_{1}$, as

$$
\left(1-\varepsilon_{\mathbf{n}}\right)(1-\varepsilon) \leq \operatorname{Av}_{\Xi_{\alpha}^{t}}\left(\left\langle\frac{\mid\left\{l: n_{k}^{t} \leq l<n_{k+1}^{t} \text { and } r_{l}^{\mathbf{n}} \in G_{P_{\alpha}}\right\} \mid}{n_{k+1}^{t}-n_{k}^{t}}: k<\omega\right\rangle\right)
$$

and $\varepsilon>0$ was arbitrary, clearly

$(*)_{\mathbf{n}} \quad$ in $\mathbf{V}^{P_{\alpha}}$,

$\sqrt{2 \varepsilon_{\mathbf{n}}} \geq \Xi_{\alpha}^{t}\left(\left\{k<\omega: 1-\sqrt{2 \varepsilon_{\mathbf{n}}} \geq \frac{\mid\left\{l: n_{k}^{t} \leq l<n_{k+1}^{t} \text { and } r_{l}^{\mathbf{n}} \in G_{P_{\alpha}}\right\} \mid}{n_{k+1}^{t}-n_{k}^{t}}\right\}\right)$.

Let

$$
A_{\varepsilon^{*}}^{\prime}=\left\{k<\omega: \text { if } \zeta \in\left[s_{k}, s_{k+1}\right) \text { and } i \in v_{0} \text { then } p_{\zeta} \backslash\left\{\gamma_{i}^{\zeta}\right\} \in G_{P_{\alpha}}\right\} .
$$

Clearly $\Xi_{\alpha}^{t}\left(A_{\sim}^{\prime} \varepsilon^{*}\right)=1$. Let $\varepsilon^{*}<1-\sum_{\mathbf{n}} \sqrt{2 \varepsilon_{\mathbf{n}}}$ and $\varepsilon^{*}>0$, so 


$$
\begin{aligned}
& {\underset{\sim}{\varepsilon^{*}}} \cup\left(\omega \backslash A_{\varepsilon^{*}}^{\prime}\right) \\
& =\left\{k<\omega: \varepsilon^{*}<\frac{\mid \bigcap_{\mathbf{n} \in v_{1}}\left\{l \in\left[n_{k}^{t}, n_{k+1}^{t}\right) \text { and } p_{\zeta}^{\prime}\left(\gamma_{\mathbf{n}}^{\zeta}\right) \in G_{Q_{\gamma_{\mathbf{n}}}}\right\} \mid}{n_{k+1}^{t}-n_{k}^{t}}\right\} \\
& \supseteq\left\{k<\omega: \text { if } \mathbf{n} \in v_{1} \text { then } \frac{\mid\left\{l: n_{k}^{t} \leq l<n_{k+1}^{t} \text { and } r_{l}^{\mathbf{n}} \in G_{P_{\alpha}}\right\} \mid}{n_{k+1}^{t}-n_{k}^{t}} \geq 1-\sqrt{2 \varepsilon_{\mathbf{n}}}\right\} \\
& =\omega \backslash \bigcup_{\mathbf{n} \in v_{1}}\left\{k<\omega: \frac{\mid\left\{l: n_{k}^{t} \leq l<n_{k+1}^{t} \text { and } r_{l}^{\mathbf{n}} \in G_{P_{\alpha}}\right\} \mid}{n_{k+1}^{t}-n_{k}^{t}}<1-\sqrt{2 \varepsilon_{\mathbf{n}}}\right\},
\end{aligned}
$$

hence $\Xi_{\alpha}^{t}\left(\sim_{\varepsilon^{*}}^{A_{\varepsilon^{*}}} \cup\left(\omega \backslash \underset{\sim}{A_{\varepsilon^{*}}^{\prime}}\right)\right) \geq 1-\sum_{\mathbf{n} \in v} \sqrt{2 \varepsilon_{\mathbf{n}}} \geq \varepsilon^{*}>0$; but

$$
\Xi_{\sim}^{\Xi}\left(\omega \backslash \underset{\sim}{A_{\varepsilon^{*}}}\right)=1-\underset{\sim}{\Xi_{\alpha}}\left(\underset{\sim}{A_{\varepsilon^{*}}^{\prime}}\right)=1-1=0
$$

and hence necessarily $A_{\varepsilon^{*}}$ is infinite.

This suffices for 3.4 .

Continuation of the proof of 3.3. Let $p^{\otimes}$ be as in Claim 3.4, let $G_{P_{\alpha}}$ be a generic subset of $P_{\alpha}$ to which $p^{\otimes}$ belongs and we shall work in $\mathbf{V}\left[G_{P_{\alpha}}\right]$. So $A=\underset{\sim}{A} \varepsilon_{\varepsilon^{*}}[G]$ is infinite. For $k \in A$, let

$$
b_{k}=\left\{\zeta \in\left[s_{k}, s_{k+1}\right): p_{\zeta}^{\prime} \in G_{P_{\alpha}}\right\} .
$$

We know that $\left|b_{k}\right|>\left(s_{k+1}-s_{k}\right) \varepsilon^{*}$. Note that if $k \in A$, then $T \cap{ }^{j_{k}} 2 \subseteq$ $\bigcap_{\zeta \in b_{k}} a_{\zeta}$ as $\lg \left(s^{*}\right)=m^{*}>m$. To reach a contradiction it is enough to show that for infinitely many $k \in A$ there is a bound on the size of $c_{k}=T \cap{ }^{j_{k}} 2$ which does not depend on $k$.

Now $\left|b_{k}\right| /\left(s_{k+1}-s_{k}\right)$ is at most the probability that if we choose a subset of ${ }^{\left(j_{k}\right)} 2$ with $2^{j_{k}}\left(1-8^{-m^{*}}\right)$ elements, it will include $T \cap{ }^{\left(j_{k}\right)} 2$; now if $k \in A$ this probability has a lower bound $\varepsilon^{*}$ not depending on $k$, and this implies a bound on $\left|T \cap{ }^{\left(j_{k}\right)} 2\right|$ not depending on $k$. More formally, for a fixed $k<\omega$ we have

$$
\begin{aligned}
\left|b_{k}\right| & =\left|\left\{a_{\zeta}: \zeta \in\left[s_{k}, s_{k+1}\right), \zeta \in b_{k}\right\}\right| \\
& \leq\left|\left\{a_{\zeta}: \zeta \in\left[s_{k}, s_{k+1}\right), T \cap{ }^{\left(j_{k}\right)} 2 \subseteq a_{\zeta}\right\}\right| \\
& \leq \mid\left\{a \subseteq{ }^{\left(j_{k}\right)} 2: T \cap{ }^{\left(j_{k}\right)} 2 \subseteq a \text { and }|a|=2^{j_{k}}\left(1-8^{-m^{*}}\right)\right\} \mid \\
& =\left|\left\{a \subseteq{ }^{\left(j_{k}\right)} 2 \backslash\left(T \cap{ }^{\left(j_{k}\right)} 2\right):|a|=2^{j_{k}} 8^{-m^{*}}\right\}\right| \\
& =\left(\begin{array}{c}
2^{j_{k}}-\left|T \cap \cap^{\left(j_{k}\right)} 2\right| \\
2^{j_{k}} 8^{-m^{*}}
\end{array}\right) .
\end{aligned}
$$

Similarly

$$
s_{k+1}-s_{k}=\left(\begin{array}{c}
2^{j_{k}} \\
2^{j_{k}} 8^{-m^{*}}
\end{array}\right) .
$$


Let $i_{k}(*)=\min \left\{\left|T \cap{ }^{\left(j_{k}\right)} 2\right|, 2^{j_{k}-3 m^{*}}\right\}$. Hence

$$
\begin{aligned}
\left|b_{k}\right| /\left(s_{k+1}-s_{k}\right) & \leq\left(\begin{array}{c}
2^{j_{k}}-\left|T \cap{ }^{\left(j_{k}\right)} 2\right| \\
2^{j_{k}} 8^{-m^{*}}
\end{array}\right) /\left(\begin{array}{c}
2^{j_{k}} \\
2^{j_{k}} 8^{-m^{*}}
\end{array}\right) \\
& \leq\left(\begin{array}{c}
2^{j_{k}}-i_{k}(*) \\
2^{j_{k}} 8^{-m^{*}}
\end{array}\right) /\left(\begin{array}{c}
2^{j_{k}} \\
2^{j_{k}} 8^{-m^{*}}
\end{array}\right) \\
& =\prod_{i<i_{k}(*)}\left(2^{j_{k}}-2^{j_{k}} 8^{-m^{*}}-i\right) / \prod_{i<i_{k}(*)}\left(2^{j_{k}}-i\right) \\
& =\prod_{i<i_{k}(*)}\left(1-\frac{2^{j_{k}} 8^{-m^{*}}}{2^{j_{k}}-i}\right) .
\end{aligned}
$$

So (recall $k \in A$ )

$$
\begin{aligned}
\varepsilon^{*} \leq \frac{\left|b_{k}\right|}{s_{k+1}-s_{k}} & \leq \prod_{i<i_{k}(*)}\left(1-\frac{2^{j_{k}} 8^{-m^{*}}}{2^{j_{k}}-i}\right) \\
& \leq \prod_{i<i_{k}(*)}\left(1-\frac{2^{j_{k}} 8^{-m^{*}}}{2^{j_{k}}}\right)=\left(1-8^{-m^{*}}\right)^{i_{k}(*)} .
\end{aligned}
$$

So we can find a bound on $i_{k}(*)$ not depending on $k$ :

$$
i_{k}(*) \leq \log \left(1 / \varepsilon^{*}\right) / \log \left(1 /\left(1-8^{-m^{*}}\right)\right),
$$

remember $m^{*}>10$ so $1-8^{-m^{*}} \in(0,1)_{\mathbb{R}}$. So for $k$ large enough,

$$
2^{j_{k}-3 m^{*}}<\log \left(1 / \varepsilon^{*}\right) / \log \left(1 /\left(1-8^{-m^{*}}\right)\right),
$$

hence if $\left|T \cap{ }^{\left(j_{k}\right)} 2\right| \leq 2^{j_{k}-3 m^{*}}$ we are done. Otherwise, by the choice of $i_{k}(*)$,

$$
\left|T \cap{ }^{\left(j_{k}\right)} 2\right|=i_{k}(*) \leq \log \left(1 / \varepsilon^{*}\right) / \log \left(1 /\left(1-8^{-m^{*}}\right)\right) .
$$

This finishes the proof.

TheOREM 3.5. Under Hypothesis 2.1 there is $\bar{Q} \in \mathcal{K}^{3}$ with $\lg (\bar{Q})=\chi$ $=\delta^{*}$ (if clause $(\alpha)$ of 2.1 (b) holds) or $\lg (\bar{Q})=\chi \times \chi \times \lambda^{+}$(if clause $(\beta)$ of 2.1(b) holds) such that in $\mathbf{V}^{P_{\lim \bar{Q}}}$ we have $\mathrm{MA}_{<\kappa}+\operatorname{cov}($ null $)=\lambda$.

P r o of. First assume clause $(\alpha)$ of 2.1(b). By 2.3(2) and 2.3(6) it suffices to find an iteration

$$
\left\langle P_{\alpha}, \underline{Q}_{\beta}, A_{\beta}, \mu_{\beta}, \tau_{\beta}, \eta_{\beta},\left({\underset{\sim}{\Xi}}_{\alpha}^{t}\right)_{t \in \mathcal{T}}: \alpha \leq \chi, \beta<\chi\right\rangle \in \mathcal{K}^{3}
$$

(see Definition 2.11) satisfying clauses $(\mathrm{a})+(\mathrm{b})+(\mathrm{c})$ of $2.3(2)+(6)$ (as the only property missing, $\operatorname{cov}($ null $) \leq \lambda$, holds by $2.7+3.3)$.

Let $\mathcal{K}_{3}^{-}=\left\{\bar{Q} \in \mathcal{K}_{3}: \lg (\bar{Q})<\chi\right\}$.

Now choose $\bar{Q}^{\xi} \in \mathcal{K}_{3}^{-}$for $\xi<\chi$ increasing with $\xi$ (see Definition 2.12) by induction on $\chi$. If $\operatorname{cf}(\xi)>\aleph_{0}$ use $2.13(3)$, and if $\operatorname{cf}(\xi)=\aleph_{0}$ use 2.14. So we deal with $\xi+1$. Bookkeeping gives us sometimes a case $Q$ of $2.3(6)$ (c) as assignment; we can find suitable $A \subseteq \lg \left(\bar{Q}^{\xi}\right)$ by 2.3(4) and then apply 
2.19 to get $\bar{Q}^{\xi+1}$. For other $\xi$, bookkeeping gives us a case of $2.3(2)$ (b) as assignment $A \subseteq \lg \left(\bar{Q}^{\xi}\right)$ such that $|A|<\lambda$. Now we apply 2.16(2) (with $\bar{Q}$, $A$ there standing for $\bar{Q}^{\xi}, A$ here) and get $A^{\prime}$ as there. Now apply 2.16(1) with $\bar{Q}^{\prime}, A^{\prime}$ here standing for $\bar{Q}, A^{\prime}$ here standing for $\bar{Q}, A$ there (and $\eta$ any member of ${ }^{\kappa} 2 \backslash\left\{\eta_{\beta}: \beta<\lg \left(\bar{Q}^{\prime}\right)\right\}$, possible as $\lg \left(\bar{Q}^{\prime}\right)<\chi$ as $\left.\bar{Q}^{\prime} \in \mathcal{K}^{3}\right)$ and get $\bar{Q}^{\xi+1}$ (corresponding to $\bar{Q}^{+}$there).

Second, assume clause $(\beta)$ of $(b)$. We just should be more careful in our bookkeeping, particularly at the beginning let $\left\langle\eta_{\alpha}: \alpha<\chi \times \chi \times \lambda^{+}\right\rangle$be an enumeration of ${ }^{\kappa} 2$ with no repetition and note $\operatorname{cf}\left([\chi]^{<\lambda}, \subseteq\right)=\chi$ suffices instead of $\chi=\chi^{\lambda}$.

\section{References}

[Ba88] T. Bartoszyński, On covering of real line by null sets, Pacific J. Math., 131 (1988), 1-12.

[BaJu95] T. Bartoszyński and H. Judah, Set Theory: On the Structure of the Real Line, A K Peters, Wellesley, MS, 1995.

[Fe94] D. Fremlin, Problem list, circulated notes, 1994.

[Ko] P. Komjáth, On second-category sets, Proc. Amer. Math. Soc. 107 (1989), 653-654.

[Mi82] A. W. Miller, A characterization of the least cardinal for which the Baire category theorem fails, Proc. Amer. Math. Soc. 86 (1982), 498-502.

[Sh 538] S. Shelah, Historic iteration with $\aleph_{\varepsilon}$-support, Arch. Math. Logic, accepted.

[Sh 619] -, The null ideal restricted to a non-null set may be saturated, preprint.

Institute of Mathematics

The Hebrew University of Jerusalem

91904 Jerusalem, Israel

E-mail: shelah@math.huji.ac.il
Department of Mathematics Rutgers University

New Brunswick, NJ 08854, U.S.A.

URL: http://www.math.rutgers.edu/ shelah

Received 25 May 1996;

in revised form 17 June 1999 